\title{
Quantum optics and nano-optics teaching laboratory for the undergraduate curriculum: teaching quantum mechanics and nano- physics with photon counting instrumentation
}

\section{Svetlana Lukishova}

Svetlana G. Lukishova, "Quantum optics and nano-optics teaching laboratory for the undergraduate curriculum: teaching quantum mechanics and nanophysics with photon counting instrumentation," Proc. SPIE 10452, 14th Conference on Education and Training in Optics and Photonics: ETOP 2017, 104522I (16 August 2017); doi: 10.1117/12.2269872

SPIE Event: 14th Conference on Education and Training in Optics and Photonics, ETOP 2017, 2017, Hangzhou, China 


\title{
Quantum optics and nano-optics teaching laboratory for the undergraduate curriculum: teaching quantum mechanics and nano-physics with photon counting instrumentation
}

\author{
Svetlana G. Lukishova \\ The Institute of Optics, University of Rochester, Rochester NY, USA \\ E-mail: sluk@lle.rochester.edu
}

\begin{abstract}
At the Institute of Optics, University of Rochester (UR), we have adapted to the main challenge (the lack of space in the curriculum) by developing a series of modular 3-hour experiments and 20-min-demonstrations based on technical elective, 4-credit-hour laboratory course "Quantum Optics and Nano-Optics Laboratory" (OPT 253/OPT453/PHY434), that were incorporated into a number of required courses ranging from freshman to senior level. Rochester Monroe Community College (MCC) students also benefited from this facility that was supported by four NSF grants. MCC students carried out two 3-hour labs on photon quantum mechanics at the UR. Since 2006, total 566 students passed through the labs with lab reports submission (including $144 \mathrm{MCC}$ students) and more than 250 students through lab demonstrations. In basic class OPT 253, four teaching labs were prepared on generation and characterization of entangled and single (antibunched) photons demonstrating the laws of quantum mechanics: (1) entanglement and Bell's inequalities, (2) single-photon interference (Young's double slit experiment and Mach-Zehnder interferometer), (3) confocal microscope imaging of single-emitter (colloidal nanocrystal quantum dots and NV-center nanodiamonds) fluorescence within photonic (liquid crystal photonic bandgap microcavities) or plasmonic (gold bowtie nanoantennas) nanostructures, (4) Hanbury Brown and Twiss setup. Fluorescence antibunching from nanoemitters. Students also carried out measurements of nanodiamond topography using atomic force microscopy and prepared photonic bandgap materials from cholesteric liquid crystals. Manuals, student reports, presentations, lecture materials and quizzes, as well as some NSF grants' reports are placed on a website http://www.optics.rochester.edu/workgroups/lukishova/QuantumOpticsLab/ . In 2011 UR hosted 6 professors from different US universities in three-days training of these experiments participating in the Immersion Program of the Advanced Laboratory Physics Association.
\end{abstract}

Keywords: Quantum and nano-optics teaching experiments, collaboration between the university and local community college, photon quantum mechanics.

\section{INTRODUCTION}

Quantum mechanics is one of the most challenging topics of modern physics in science and engineering education, but it is now being applied to important technological problems. Enormously powerful computers and total communication security are the future goals of quantum information technology which is emerging in the market. It is important to familiarize the future workforce with these new ideas as well as to provide them with hands-on experience in photoncounting instrumentation widely used in many technological areas (e.g., nanotechnology and biomedicine). See Figure 1 for applications of photon counting technique. The goal of our many years project was to reduce to practice some of the most abstract components of quantum mechanics by allowing the students to carry out experiments at a range of levels connected with modern applications, in particular, quantum computing and quantum communication. Learning the abstract theory from hands-on experiments with modern silicon photodetectors, CCD-cameras, and computer cards provides students not only a better understanding of "quantum weirdness" but also a unique experience using state-of-the-art technology which they will encounter in their future workplace. This project was developed in a collaboration with Rochester Monroe Community College (MCC), Prof. P. D'Alessandris.

University of Rochester (UR) built four teaching experimental setups in quantum and nano-optics located in three separate rooms of the Institute of Optics with total 587 sq. ft. and taught these labs for 12 years, starting in 2006 [1]. Several universities learned UR experience in these quantum and nano-optics teaching experiments, for instance:

14th Conference on Education and Training in Optics and Photonics: ETOP 2017, edited by Xu Liu,

Xi-Cheng Zhang, Proc. of SPIE Vol. 10452, 104522I · @ 2017 ICO, IEEE, OSA, SPIE

CCC code: $0277-786 X / 17 / \$ 18 \cdot$ doi: $10.1117 / 12.2269872$ 
- In August 2011, participating in the Immersion Program of the Advanced Laboratory Physics Association (http://www.advlab.org/imm_singlephotonr2.html), six professors from different universities visited UR for 3 days to learn advanced lab experience at the Quantum- and Nano-Optics Lab facility at the UR;

- During Fall 2009, a professor from Rochester Institute of Technology (RIT) spent his sabbatical at the UR labs. Later RIT established their own quantum-optics teaching lab.

- Five students of Adelphi University and their professor visited UR labs and carried out all labs of an OPT 253 class during 1.5 days visit in 2012.

Four US National Science Foundation (NSF) grants (one Material Research Instrumentation (MRI) and 3 educational) created the basis for this work:

- MRI: Development of single photon generation and characterization unit, 09/15/2004-08/31/2007;

- Course, Curriculum, Laboratory Improvement (CCLI)-Phase I: Quantum optics laboratory for the undergraduate curriculum - teaching quantum mechanics with photon counting instrumentation (collaboration with MCC), 06/05/200705/31/2010;

- Collaborative research - CCLI-Phase II: Diverse partnership in teaching quantum mechanics and modern physics with photon counting instrumentation (collaboration with MCC, RIT and Bryn Mawr College), 09/05/2009-08/31/2013;

- Nanotechnology Undergraduate Education (NUE) in Engineering: Development of multidisciplinary nanotechnology undergraduate education program at the University of Rochester Integrated Nanosystems Center (collaboration with MCC), 01/01/14-12/31/16.

The Institute of Optics teaching laboratories, the Spectra-Physics division of Newport Corporation, and two University of Rochester (UR) grants also supported this program:

- UR Kauffman Foundation: Entanglement to entrepreneurship: how do fundamental scientific advances spawn new business, 01/01/2006-12/31/2006;

- Wadsworth C. Sykes Faculty Engineering Award, the Hajim School of Engineering and Applied Sciences: Developing quantum optics and nano-optics teaching laboratory for undergraduates, 04/01/2012 -03/31/2013.

Four labs were prepared on generation and characterization of entangled and single photons demonstrating the laws of quantum mechanics: (1) entanglement and Bell's inequalities; (2) single-photon interference (Young's double slit experiment and Mach-Zehnder interferometer); (3) single-photon source: confocal microscope imaging of single-emitter fluorescence; (4) single-photon source: Hanbury Brown and Twiss setup. Fluorescence antibunching. From these labs a 4-credit hour technical elective course OPT 253/OPT 453/PHY 434 "Quantum and Nano-Optics Laboratory" was prepared and taught from Fall 2006. Manuals, student reports, presentations and lecture materials are placed on a website [1]. Both undergraduate and graduate versions of this class was developed. OPT 453 became a required class for a Master's in Optics degree with a specialty Nano- and Integrated Photonics.

Some labs of this course were introduced to the following UR courses:

- "Quantum Mechanics of Optical Materials and Devices" OPT 223 (two 3-hour labs);

- "Introduction to Optics ", a freshmen course OPT 101 (12-hour research projects on one of the four labs);

- "Sources and Detectors Labs" OPT 204 (one 3-hour lab);

- "Nanometrology Laboratory" OPT 254 (one 12-hour lab);

- “Advanced Experimental Techniques I" PHY 243W (one 12-hour lab);

- "Advanced Topics in Quantum Optics" PHY 534/OPT 554 (two 6-hour labs).

Monroe Community college students of the course "Modern Physics" carried out these labs at the UR (two 3-hour labs).

This paper is organized as follows. Section 2 describes four teaching experiments of a Quantum and Nano-Optics Laboratory class. Section 3 outlines freshman research projects and addition of quantum labs to some undergraduate and graduate classes at the UR. Section 4 discusses teaching students and professors from other universities and colleges at the UR Quantum and Nano-Optics Lab facility. Section 5 presents some methods of evaluation of students' knowledge. Section 6 concludes the paper. 


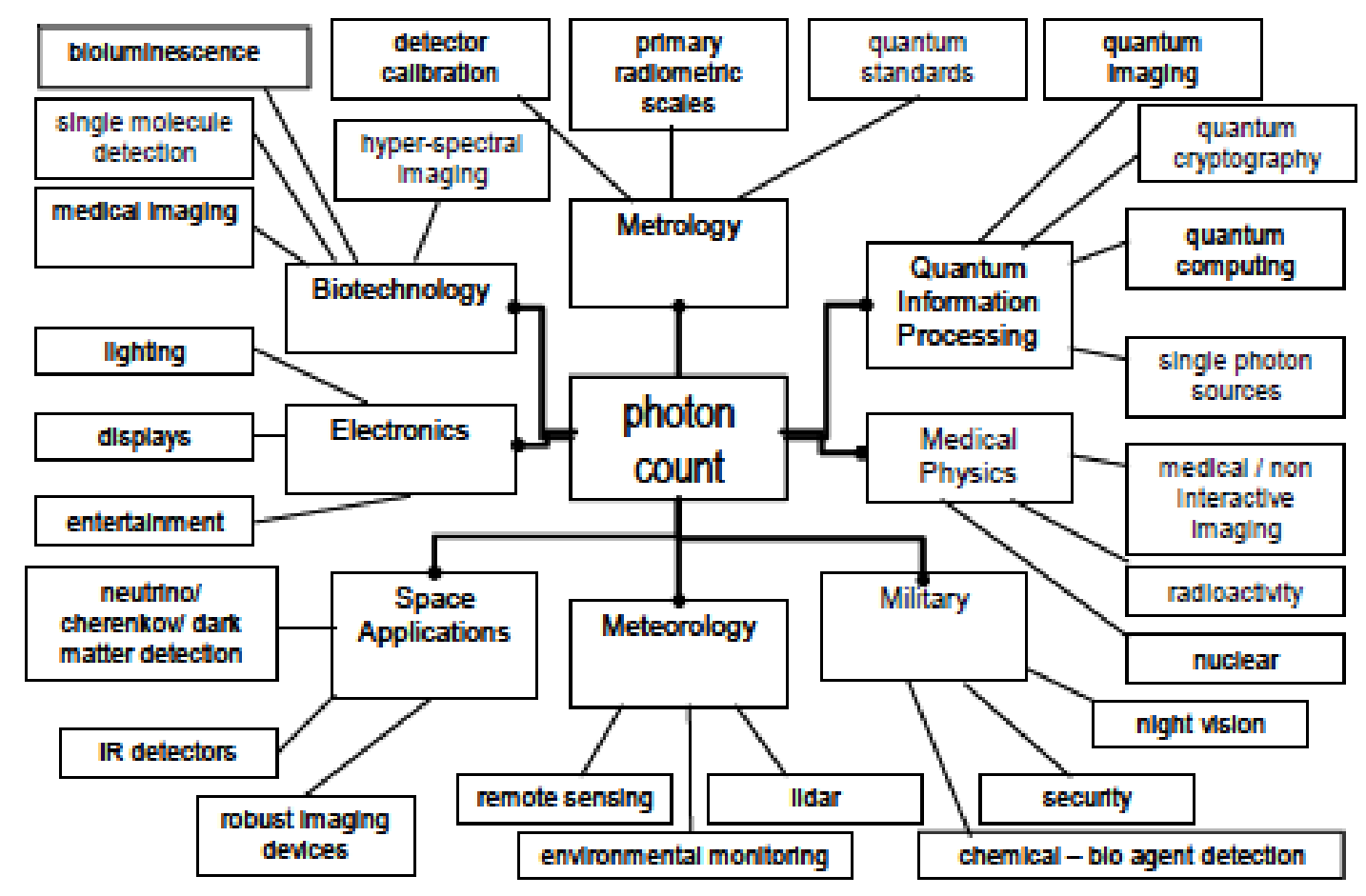

Figure 1. Areas of applications of photon counting instrumentation (from the second international workshop "Single Photon: Sources, Detectors, Applications and Measurements Methods" (Teddington, UK, 24-26 October 2005).

\section{DESCRIPTION OF PREPARED TEACHING EXPERIMENTS}

\subsection{Lab. 1. Entanglement and Bell's inequalities}

Entanglement is the most exciting and mysterious property of some quantum mechanical systems when property of one particle depends on the property of the other. Measurements performed on a first particle would change the state of the second particle, no matter how far apart they may be. This nonlocal character is a key in entanglement and it is important that no propagation of information occurs. Entanglement can be on some physical value, e.g., polarization, energy, momentum, time, etc. Mathematically we can say that in quantum mechanics, particles are called entangled if their state cannot be factored into single-particle states:

$$
\left|\Psi_{12}\right\rangle \neq\left|\Psi_{1}\right\rangle \otimes\left|\Psi_{2}\right\rangle
$$

Figure 2 shows a cartoon made by A.K. Jha and L. Elgin showing "entangled particles". Applications of quantum entanglement are quantum computers, quantum communication, and quantum teleportation (teleportation of a state, but not a particle).

The idea of entanglement was introduced into physics in 1935 by Einstein, Podolsky and Rosen (EPR) [2], as "spooky action at a distance" (entanglement), in which authors (EPR) did not believe, because of information cannot propagate with the speed greater than the speed of light. EPR also did not like probabilities in description of particle behavior. They suggested, that quantum mechanics is incomplete theory, and future, complete theory of quantum mechanics will be something like statistical mechanics with some

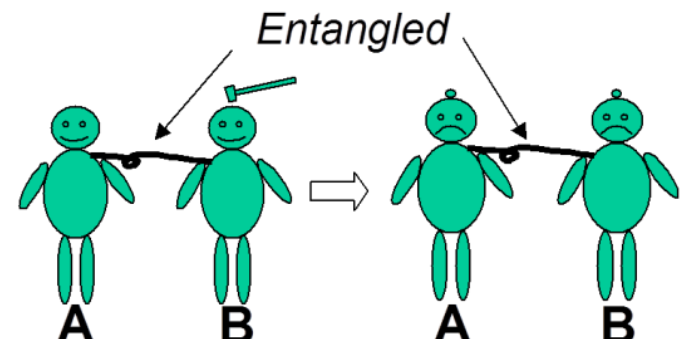

Figure 2. Cartoon showing "entangled particles". 
additional variables which later have been named "hidden". Shortly after the EPR paper, Schrödinger coined the word entanglement (verschränkung in German) and further developed this concept [3]. Bohr replied to the EPR paradox that in a such quantum state one could not speak about the individual properties of each of the particles, even if they were distant from one another.

In the mid-sixties of the last century it was realized that the nonlocality of nature is a testable hypothesis. 1964, John Bell showed that the "locality hypothesis" with "hidden" variables leads to a conflict with quantum mechanics $[4,5]$. He proposed a mathematical theorem containing certain inequalities. An experimental violation of his inequalities would suggest the states obeying the quantum mechanics with nonlocality. I would like to emphasize that Bell's inequalities (there are many types of Bell's inequalities, some of them were obtained later by other researchers) are classical relations [6], and they are violated only in quantum mechanics and only for some values of parameters (e.g., under some polarizer angles if we have entanglement in polarization). In many values of parameters both classical physics and quantum mechanics give the same results without violation of Bell's inequalities. Quantum correlation is a very rare event, and it is not easy to find it. The most popular form of Bell's inequality for the experimentalists is a CHSH inequality described by Clauser, Horne, Shimony and Holt, in a widely-cited paper [7] published in 1969. The CHSH inequality for polarization entanglement can be obtained from a trivial relation that modulus of sum is less or equal to sum of moduli. And in a quantum world this classical relation can be violated. It is impossible to understand with a "common sense" mind, that inequality $|a+b+c| \leq|a|+|b|+|c|$ can be violated, but in this lab students violate this inequality.

The most popular way to obtain entangled photons is to use a spontaneous parametric down conversion (SPDC) process. This paper describes the lab on photons entangled in polarization. The first experiment with the same type of setup was made by Kwiat [8]. To learn how to build a similar setup see also papers [9-17], books [18, 19] and websites [1, 20, 21]. To learn about entanglement and quantum optics in general see books [22-27], and about SPDC - book of Klyshko [28].

In UR teaching lab two polarization entangled photons are produced through a SPDC in two type I Beta Barium Borate (BBO) crystals pumped by a laser. We used three experimental setups with diode lasers (405 and $408 \mathrm{~nm}$ wavelengths) and an argon ion laser (363.8 nm wavelength) with an intracavity etalon inside. In this process a single pump photon spontaneously splits into "signal" and "idler" photons with a longer wavelengths inside a nonlinear crystal. The efficiency of this process is only $\sim 10^{-10}$. Because of spontaneous process, the angles of emitted photons and their wavelength may have any values obeying the conservation of energy and momentum, so that downconverted photons, like in a rainbow, have various wavelengths. To create an entangled state at $2 \lambda$ wavelength (e.g., $727.6 \mathrm{~nm}$ for an ion-argon laser), we select definite angles of signal and idler photons' propagation and narrow-band width interference filters ( $10 \mathrm{~nm}$ bandwidth). The signal and idler photons with $2 \lambda$ wavelength are emitted in a cone (see Figure 3 ).
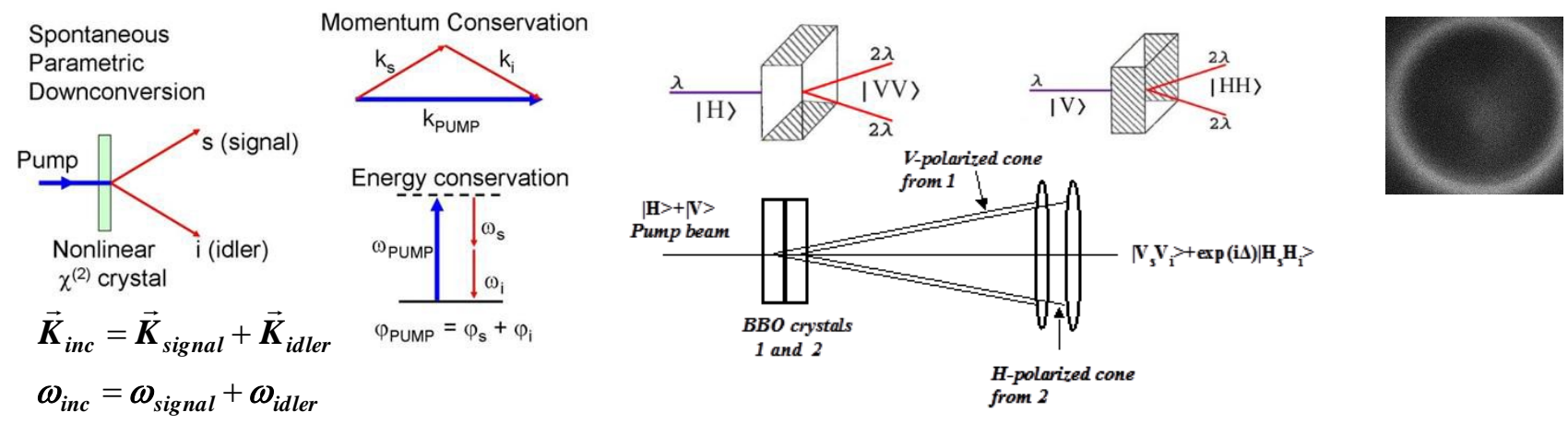

Figure 3. Experimental setup for entanglement and Bell's inequality violation. LEFT: Schematics of a SPDC process with conservation of energy and momentum; CENTER: Top - Type I SPDC: down-conversion of a horizontal photon (for this orientation of the crystal, vertical photons pass strait through) and down-conversion of a vertical photon (for this orientation of the crystal horizontal photons pass straight through). RIGHT (top): cross-section of a SPDC cone (727.6 nm wavelength) from a type I BBO crystal (measurements were made by an EM-CCD-camera).

Type I downconversion means that the signal and idler photons have the same polarization, which is opposite to that of the pump photon (Figure 3, top center). Our source of entangled photons uses two identical BBO crystals, with one rotated $90^{\circ}$ from the other about the beam propagation direction. In this arrangement each crystal can support downconversion of one pump polarization, the other polarization simply passes through the transparent crystal. A $45^{\circ}$ polarized pump photon can downconvert in either crystal, producing a polarization entangled pair of photons (Figure 3, center bottom). 
Mathematically it can be represented as $|H\rangle+|V\rangle \rightarrow\left|V_{s} V_{i}\right\rangle+\exp (i \Delta)\left|H_{s} H_{i}\right\rangle$, (2). Here $V$ and $H$ represent a horizontalor a vertical-polarized photon. Signal and idler photons are denoted by subscripts "s" and "i" respectively. $\Delta$ is a phase difference after two crystals due to different path length for different down-converted polarizations in a birefringent BBO crystal. Downconverted photons with horizontal and vertical polarizations are produced in different crystals, so they are independent from each other, and two overlapping cones of downconverted photons (Figure 3, center bottom) with vertical and horizontal polarizations give cone of unpolarized photons, although we will measure correlations in polarizations. Cross-section of a cone of downconverted photons of one $727.6 \mathrm{~nm}$ wavelength selected through a 10-nm transmission filter recorded by an EM-CCD camera is shown in the top of Figure 3, right (it was obtained with an argon ion laser).

Figure 4 shows the schematics of the experimental setup with a $405 \mathrm{~nm}$ diode laser. 10-nm bandwidth interference filters are used for selection of definite wavelength of downconverted photons $(810 \mathrm{~nm}$ in this setup) propagating at definite cone angle. Two single-photon counting avalanche photodiode modules (APDs) are used as detectors A and B. Collection system (microscope objectives and optical fibers) to APDs is located at the opposite ends of a downconverted cone diameter. See also Figure 5, left with collection system, APDs and two linear polarizers mounted on rotating mounts. The crystal set mounting for its precise alignment is shown in Figure 5, right.

Using polarizers rotated to angles $\alpha$ and $\beta$ in the signal and idler paths, respectively, one measures the polarization correlation of the downconverted photons. It is very important that response from two single-photon counting avalanche photodiode modules (APDs) was processed with a counter-timer computer card that permit to record not only signals from each APDs (singles), but also their simultaneous response (coincidences).

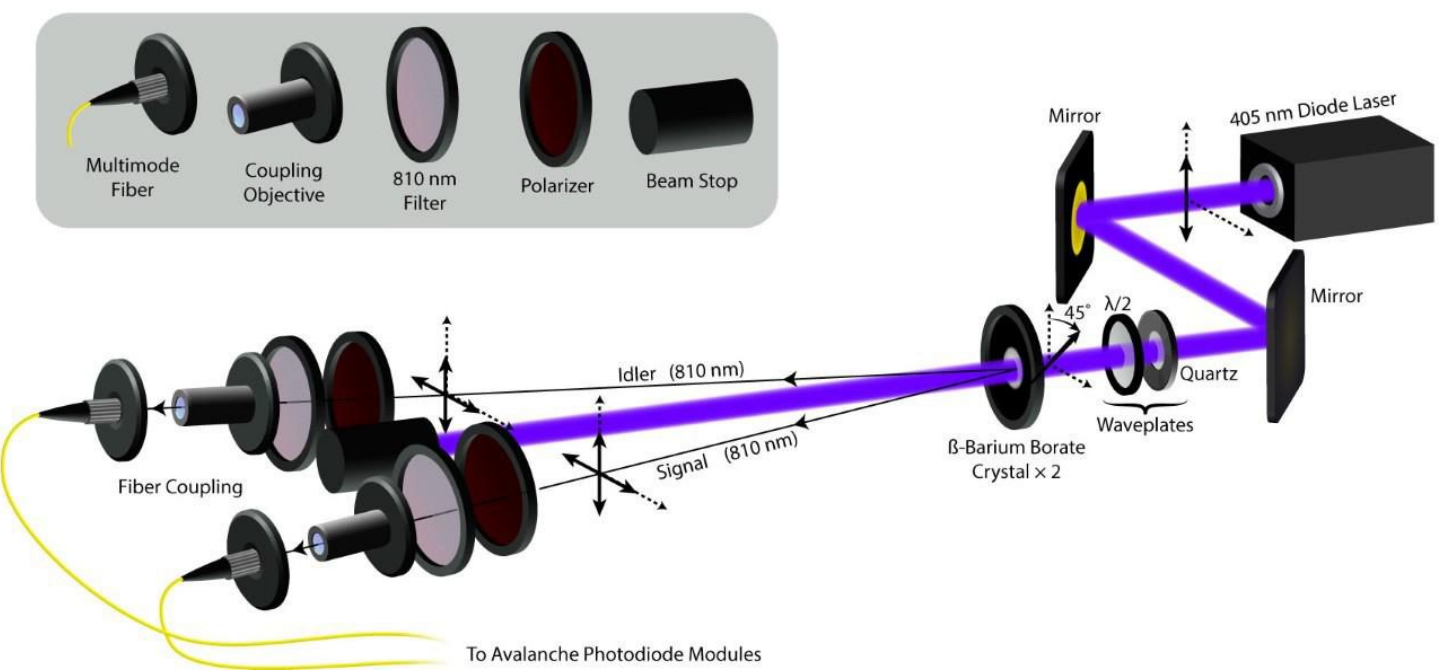

Figure 4 (prepared by R. Lopez-Rioz) shows the schematics of experimental setup of polarization entangled photons with two BBO crystals and a diode laser. We did not use a quartz plate for compensation a phase $\Delta$ to obtain entangled photons in a last version of a setup with a $405 \mathrm{~nm}$ laser, although in an OPT 253 class students investigated its effect on results by its rotation around two axes.

The probability $P$ of coincidence detection for the case of $45^{\circ}$ incident polarization on a BBO crystal set depends only on the relative polarizer angle $\beta$ - $\alpha$ :

$$
P(\alpha, \beta)=1 / 2 \cos ^{2}(\beta-\alpha) \text {. }
$$

It should be noted that APD singles' count does not depend on polarizers' rotation angles (overlapping of two cones of downconverted light from two independent polarized sources created in two different crystals results in an

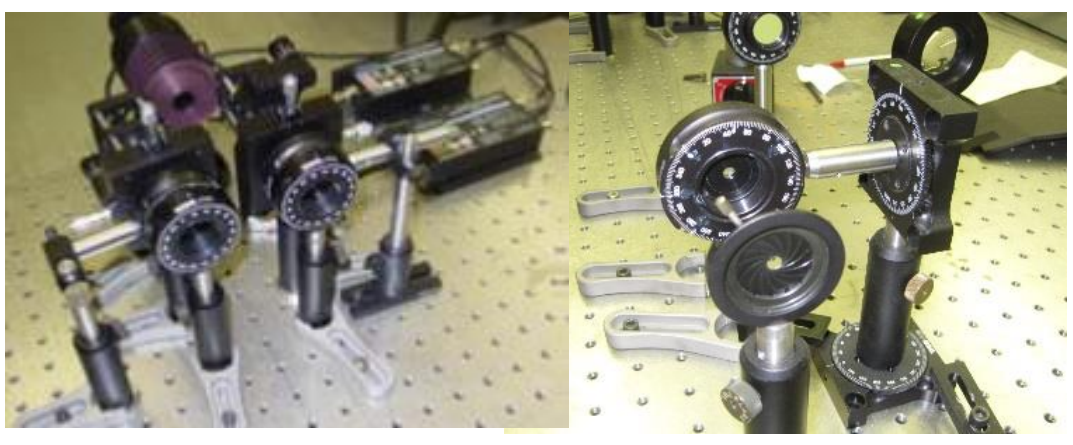
unpolarized light). The correlations described by equation (3) can be recorded only in coincidence count using a counter-timer board (time window of $25 \mathrm{~ns}$ ).

Figure 5. LEFT: Collection system with a 3-D adjustable mount with two polarizers and APD detectors. RIGHT: BBO crystal set with a 3-D rotation mounting (an iris diaphragm serves for alignment). 
The pictures of two entanglements setups with a diode $(405 \mathrm{~nm}, 120 \mathrm{~mW})$ and an ion-argon $(363.8 \mathrm{~nm}, 100 \mathrm{~mW}$, singlelongitudinal mode) lasers are shown below in Figure 6. Two fiber-connected APDs are used in both setup. BBO crystal set were cut at different angles for each setup.
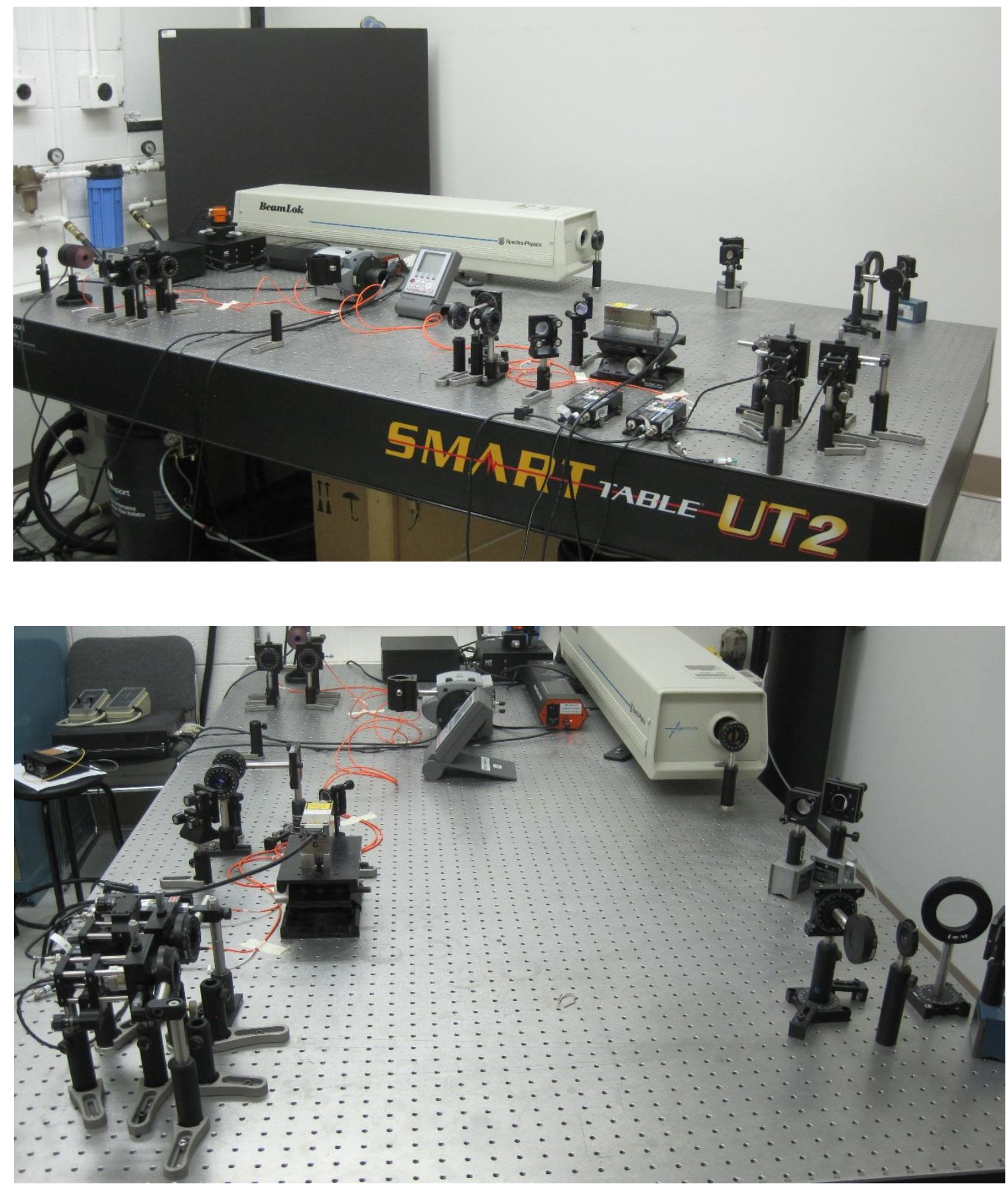

Figure 6. Two entanglement setups for the teaching labs of the Institute of Optics, University of Rochester (different views of the same optical table). 
Figure 7 shows experimental polarization correlation for fixed angles of a polarizer A $\alpha=45^{\circ}$ and $135^{\circ}$ and rotation of a polarizer B only. The experimental data are in a good agreement with relation (3): coincidence counts reach a maximum, when polarizers are parallel $(\alpha=\beta)$. Fringe visibility Vis $=\frac{I_{\max }-I_{\min }}{I_{\max }+I_{\min }}$ of 0.9 was observed that is greater than 0.71 that indicates a possibility of entanglement.

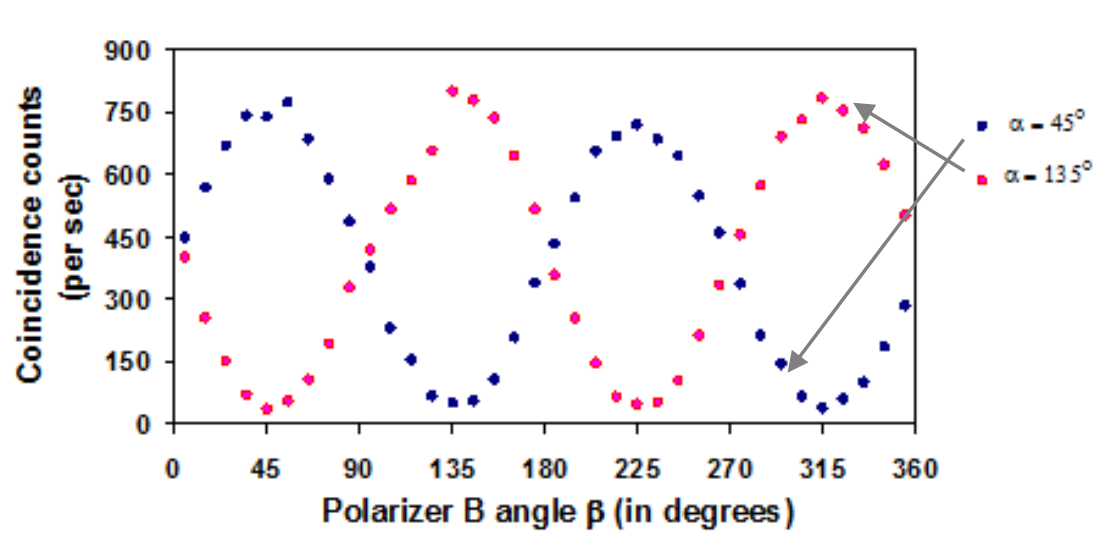

Figure 7. Polarization correlations: dependence of coincidence count on relative polarizer angle.

To prove an entanglement in our system Bell's inequality should be violated. The CHSH Bell inequality [7] constrains the degree of polarization correlation under measurements at different polarizer angles. The proof involves two measures of correlations, $E(\alpha, \beta)$ and $S\left(P_{V V}\right.$ and $P_{H H}$ are probabilities of both photons to have vertical or horizontal polarizations, $P_{V H}$ and $P_{H V}$ are probabilities of photons to have opposite polarizations), $N(\alpha, \beta)$ is measured coincidence counts at polarizers angles $\alpha$ and $\beta$. Violation of CHSH Bell inequality occurs if $\mathrm{S}>2$. $S$ does not have a clear physical meaning.

$$
\begin{aligned}
E(\alpha, \beta) & =P_{V V}(\alpha, \beta)+P_{H H}(\alpha, \beta)-P_{V H}(\alpha, \beta)-P_{H V}(\alpha, \beta), \\
E(\alpha, \beta) & =\frac{N(\alpha, \beta)+N\left(\alpha_{\perp}, \beta_{\perp}\right)-N\left(\alpha, \beta_{\perp}\right)-N\left(\alpha_{\perp}, \beta\right)}{N(\alpha, \beta)+N\left(\alpha_{\perp}, \beta_{\perp}\right)+N\left(\alpha, \beta_{\perp}\right)+N\left(\alpha_{\perp}, \beta\right)} \\
S & =E(a, b)-E\left(a, b^{\prime}\right)+E\left(a^{\prime}, b\right)+E\left(a^{\prime}, b^{\prime}\right)
\end{aligned}
$$

The above calculation of $S$ requires a total of 16 coincidence measurements $(N)$, at definite polarization angles $a$ and $b$ (see the Table 1 bellow with experimental coincidence counts). Polarization angles are selected with maximum value of $S$ from CHSH Bell's inequality to violate it. At many other angles $S$ is less than 2 from a CHSH theory, so to prove entanglement we need to select polarizer angles with max value of S. Calculation of Bell's inequality in the CHSH form

\begin{tabular}{|c|c|c|c|c|}
\hline$\beta$ & $b=-22.5^{\circ}$ & $b^{\prime}=22.5^{\circ}$ & $b_{\perp}=67.5^{\circ}$ & $b^{\prime}{ }_{\perp}=112.5^{0}$ \\
\hline$a=-45^{\circ}$ & 162 & 41 & 93 & 204 \\
\hline$a^{\prime}=0^{0}$ & 154 & 186 & 51 & 20 \\
\hline$a_{\perp}=45^{\circ}$ & 36 & 183 & 223 & 102 \\
\hline$a^{\prime} \perp=90^{\circ}$ & 56 & 57 & 278 & 267 \\
\hline
\end{tabular}
shows its violation $(S \sim 2.65>2)$.

Table 1.

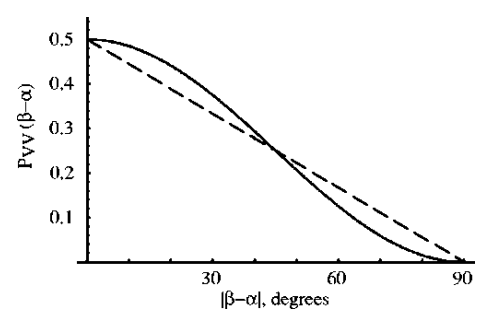

LEFT: Table 1. Data of 16 measurements of coincidence counts at 16 combinations of polarizer angle with maximum value of $S$. In this case $S>2$. At some angles, the value of $S$ can be less than 2. RIGHT: Figure 8. Dependence of $P_{\mathrm{vv}}$ on relative polarizer angles for quantum mechanical case (solid line) and for classical physics (dashed line) [10]. For some values of relative polarizer angle quantum mechanics and classical physics give the same value of $P_{\mathrm{vv}}$ as well as $S$. 


\subsection{Lab 2. Single photon interference (Young's double slit experiment and Mach-Zehnder interferometer)}

In this lab, wave-particle duality (complementarity) on example of single photons was demonstrated. For this lab an attenuated laser beam (Poissonian light source) is a good approximation for a source of single photons, although photon antibunching (separation of all photons in time) cannot be achieved in a such a source. Interference between single photons was observed in both Young's double slit and Mach-Zehnder interferometers. In a Mach-Zehnder interferometer experiment, the effect of "which path" information was also shown, since we could only acquire an inference pattern when that information was hidden by using a quantum eraser ( $45^{\circ}$ linear polarizer). This laboratory provides a visual demonstration of the appearance and disappearance of interference fringes, both at high light and single-photon levels, by carefully destroying and restoring "which-path" information using a quantum eraser in a Mach-Zehnder interferometer (see also Ref. [ 15, 29]).

Measurements are made using a He-Ne laser beam, attenuated to a single photon level with neutral density filters. On advanced lab level (OPT 253 class), cooled electron multiplying (EM) CCD camera iXon of Andor Technologies, sensitive to single-photons, is used. It was also employed with MCC students' groups and freshman research projects (OPT 101). In the OPT 204 class with 14 students' groups working at different time on the same setup (49 students total in Spring 2017), a conventional CCD camera was utilized for recording interference fringes from faint laser light attenuated for a single photon level ( one photon per meter or kilometer), but at a longer exposure time (several to tens of seconds).

Figures 9 and 10 show the schematics of two experiments of this lab: Young's double slit (Figure 9) and Mach-Zehnder interferometers (Figure 10). As a double slit we initially used $10 \mu \mathrm{m}$ width slits with $90 \mu \mathrm{m}$ slit separation fabricated by a lithographic deposition of the metal on a glass substrate. This type of a double slit provides interference pattern as a result of interference of light diffracted by the slit and reflected light from reflective surface of a substrate. In this case a fine structure appears in the interference maxima of a double slit interference (see insert in Figure 9, right). Although it does not influence a wave-particle duality, we transferred recently to the slit with opening in the air. The third part of the lab (even in a 3-hour version) is alignment of a Mach-Zehnder interferometer. A teaching assistant (TA) destroys the alignment by removing the mirror mounts from the post holders. After that students should realign an interferometer.

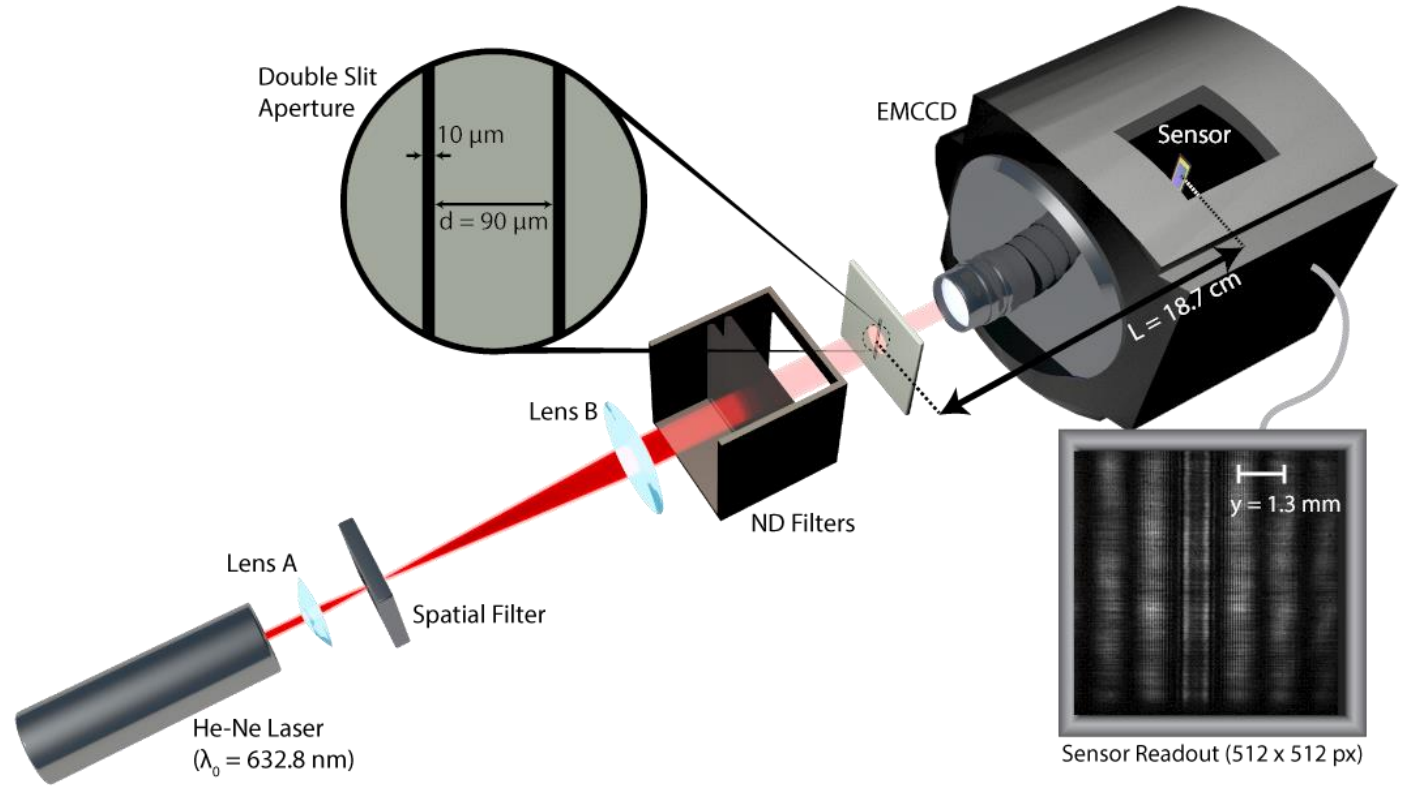

Figure 9 (prepared by R. Lopez-Rios). Setup of a single photon Young's double slit experiment. ND: Neutral density filters; EMCCD: electron multiplying CCD camera; He-Ne: Helium-Neon laser. The insert at the right shows double-slit interference with a "lithographic" slit. A fine structure is seen inside the maxima (see explanation in the text).

Figure 11 shows the results on wave-particle duality using single-photon interference pattern in a Mach-Zehnder interferometer recorded by an EM-CCD camera with a single, $200 \mathrm{~ms}$ exposure time and with accumulation of 50 images. It also shows dependence of fringe visibility of an interference pattern on the angle of quantum eraser. Figure 12 shows a single-photon interference lab setup in different views with two CCD cameras (conventional and EM-CCD).See also Ref. [23] with a similar teaching lab experiment. 


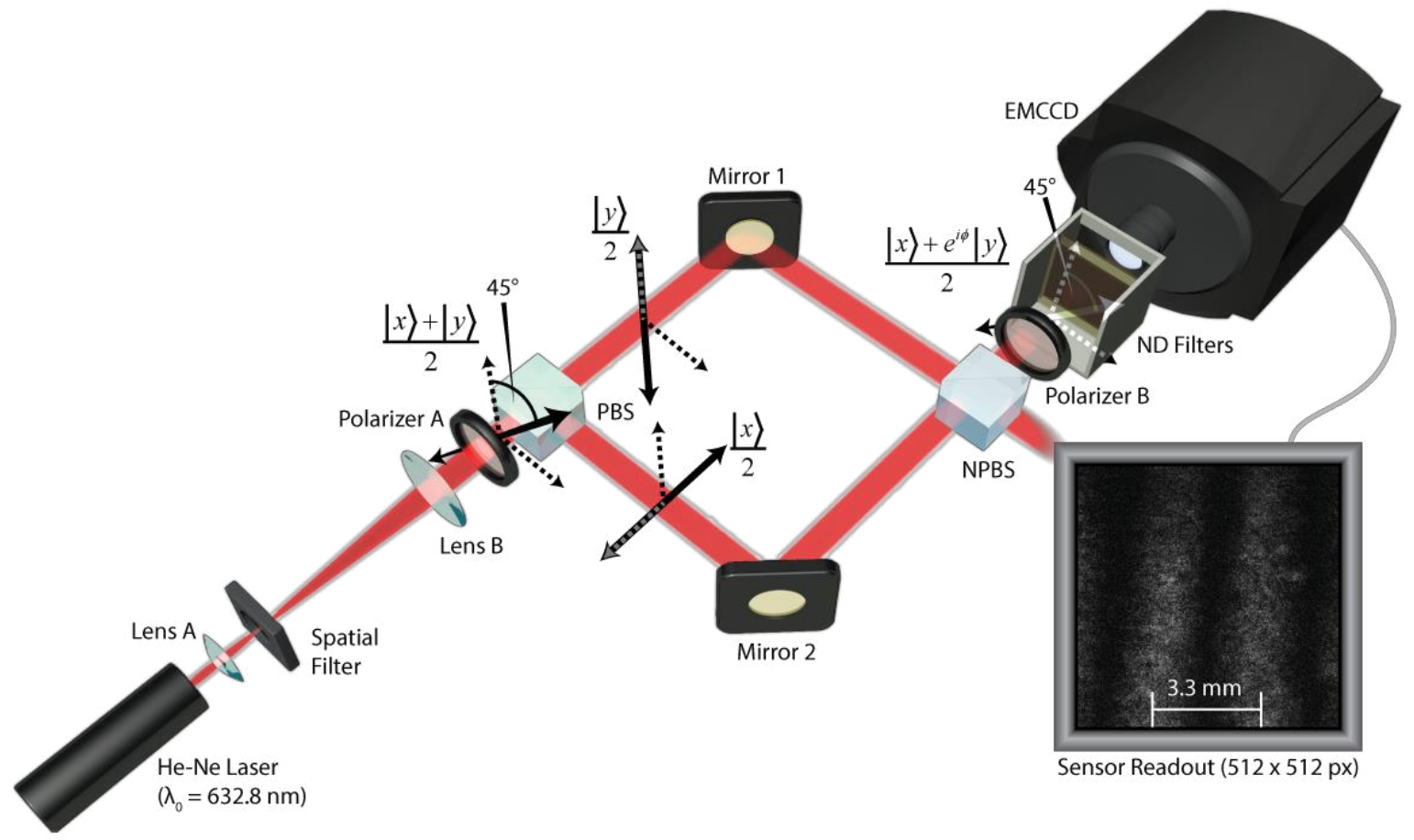

Figure 10 (prepared by R. Lopez-Rios). Setup for a Mach-Zehnder Interferometer. PBS: polarizing beam splitter; NPBS: non-polarizing beam splitter. At each point where the system state changes the polarization vector is shown.
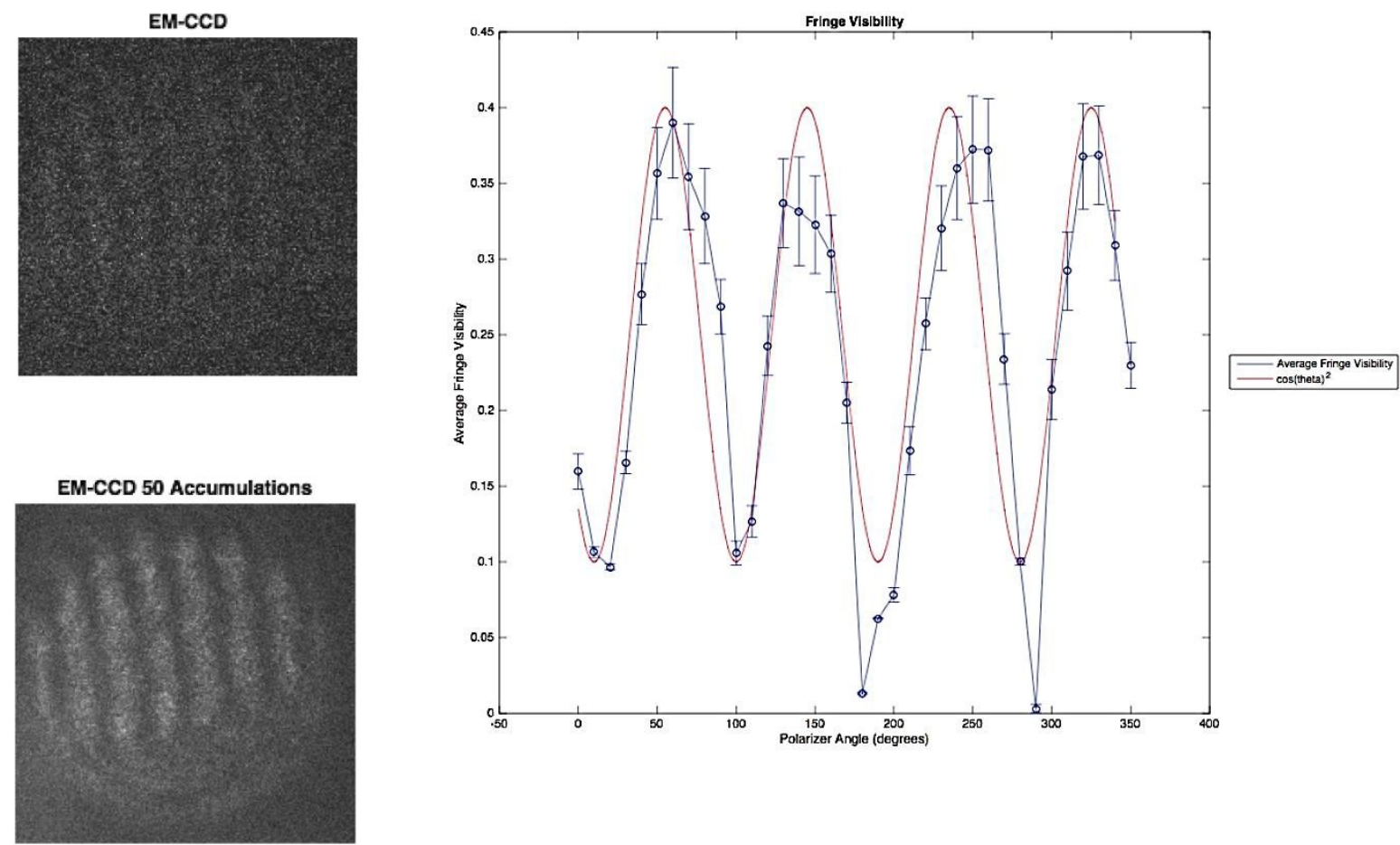

Figure 11 (from a M. Dupuis and B. Maas report, Fall 2016, OPT 453 class). LEFT: EM-CCD images of the same interference pattern at a single photon level with a $200 \mathrm{~ms}$ single exposure time (top) and with accumulation of 50 images with a $200 \mathrm{~ms}$ exposure time. RIGHT: calculation of fringe visibility of accumulated images versus a linear polarizer (quantum eraser) angle. A zero on a mount scale of a linear polarizer (quantum eraser) had s small shift relative a polarizer axis. Maximum fringe visibility should be at a $45^{\circ}$ angle. 

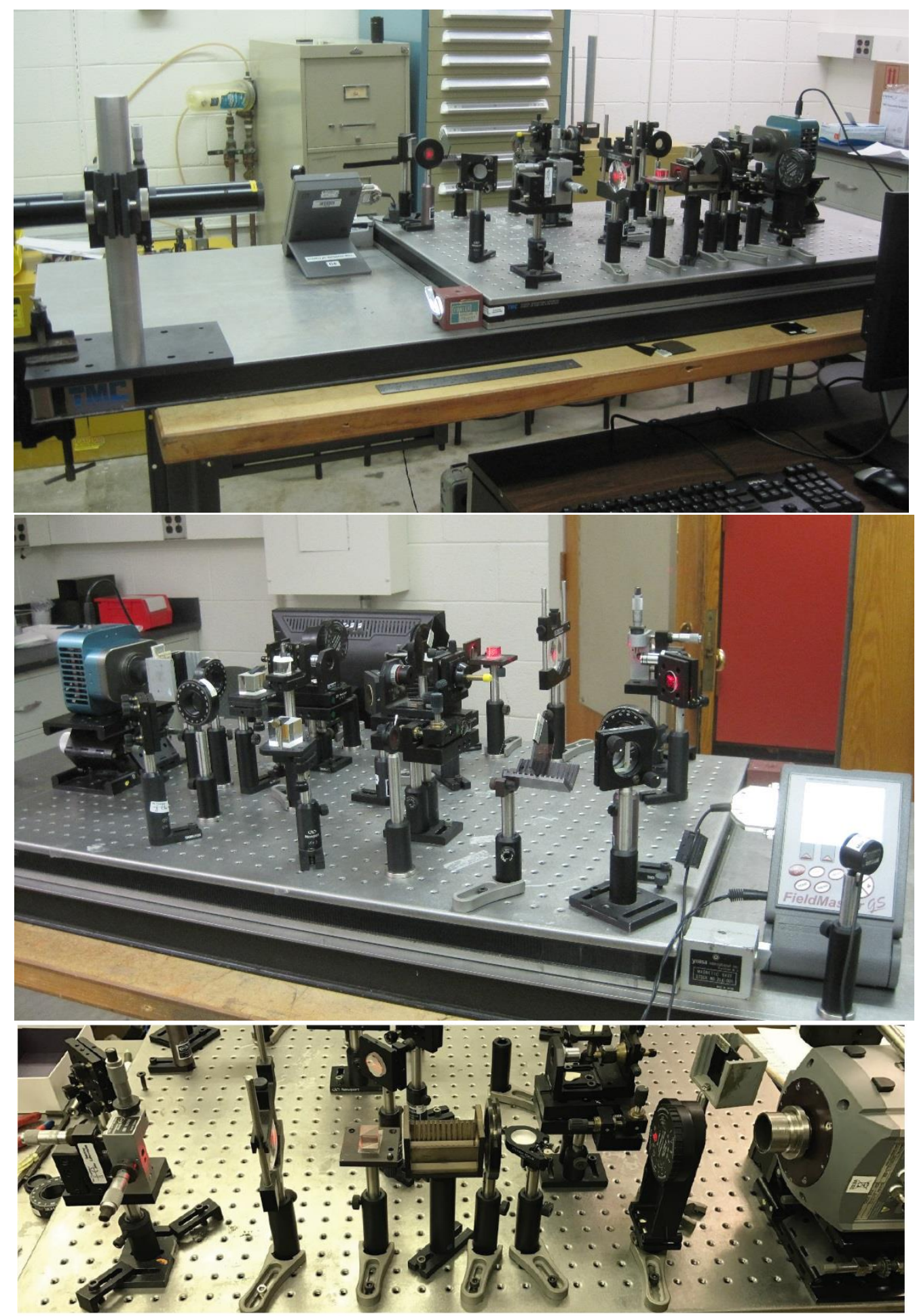

Figure 12. Experimental setup for single-photon interference lab at different viewing angles. TOP: The whole view including a HeNe laser from a Young's double slit interferometer side; CENTER: View from a Mach-Zehnder interferometer side. A conventional CCD camera without cooling and amplification of the signal is used on top and center images. BOTTOM: A Young's double slit interferometer setup with an EM-CCD camera and a slit set with air slit opening (a He-Ne laser is not shown). 


\subsection{Lab 3. Single photon source: Confocal microscope imaging of single-emitter fluorescence}

One strength of the OPT 253 course is the students' immersion in a real research environment, working on state-of-theart, fragile, and expensive equipment that modern quantum-optics research uses around the globe. Every student understands the cost of each piece of equipment he/she is entrusted with. In addition, in the Labs 3 and 4, a class time was reserved for addressing "real" research questions on actual research samples or, time permitting, have students prepare their own samples with single emitters that nobody else before them had ever investigated. This intentional blurring of the dividing line between "education" and "research" strongly boosts student interest. Occasionally students obtained results that were reported at the professional conferences, e.g. QELS, IQEC or Frontiers in Optics. Some undergraduates felt sufficiently motivated to focus their senior research projects on work in Lukishova's research lab after this class.

Labs 3 and 4 are devoted to single (antibunched) photon source (SPS) [30-31], a key hardware element in quantum cryptography. To create single photons a laser beam should be focused on a single emitter that emits single photons at a time (for reviews see [32-33]. Single colloidal semiconductor nanocrystal quantum dots and color centers in nanodiamonds were used as single emitters in these lab. A confocal fluorescence microscope (Figure 13) with excitation by the laser light with different wavelengths was used in a Lab 3 for imaging of single emitter fluorescence and measuring their spectra.

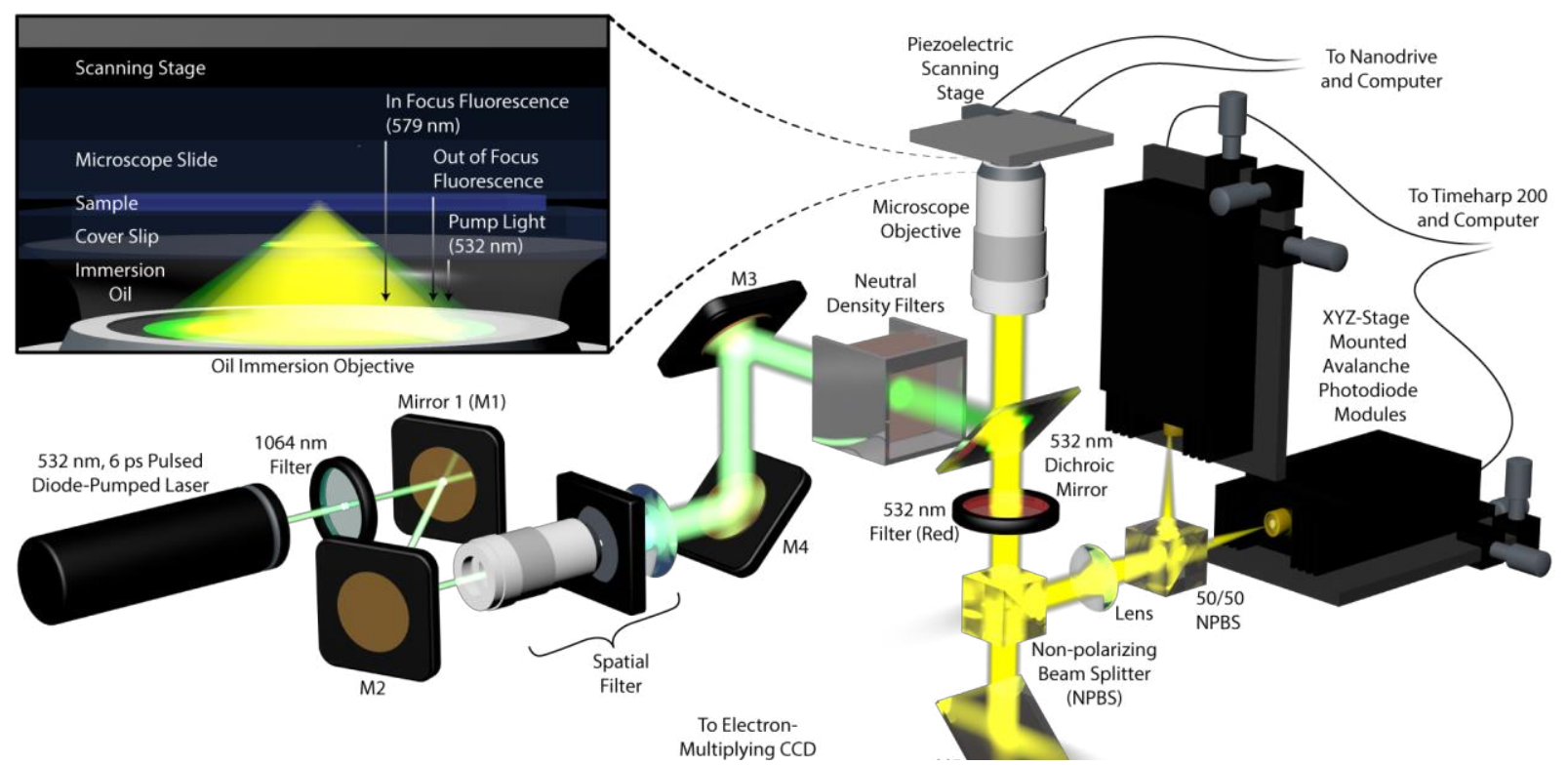

Figure 13 (prepared by R. Lopez Rios). A schematics of a confocal fluorescence microscope. Top-left inset shows oil immersion microscope objective.

Figure 14 shows confocal microscope fluorescence images of NV-color centers nanodiamonds of 40 and $20 \mathrm{~nm}$ diameter (left two images) and photoluminescence of gold bowtie nanoantenna arrays (insert shows nanoantenna shape with $75 \mathrm{~nm}$ arms and 30-60 nm gaps) (center image). Right picture shows a wide-field view of a sample area in a white light. Numbers identify the positions of nanoantenna arrays with different gaps. Students learned how to find a specific array for imaging.
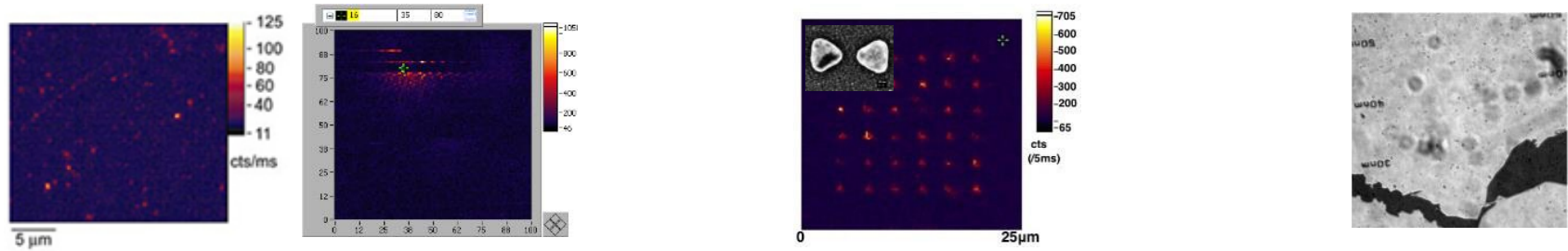

Figure 14. LEFT: Confocal microscope imaging of single NV-centers fluorescence in 40-nm and $20 \mathrm{~nm}$ size nanodiamonds. Right figure of two shows blinking in fluorescence (horizontal stripes) of a single color center in a 20-nm-nanodiamond ( $2 \mu \mathrm{m} \times 2 \mu \mathrm{m}$ raster scan). CENTER: Confocal microscope imaging of gold photoluminescence from bow-tie nanoantenna arrays. Insert shows the bow-tie shape of nanoantenna (SEM micrograph). RIGHT: A wide-field sample view recorded by a CCD-camera showing a position of different nanoantenna arrays (numbers $30 \mathrm{~nm}-50 \mathrm{~nm}$ are the gaps of nanoantennas located close to these numbers). 
Figure 15 shows the experimental setup of Labs 3 and 4 in different viewing angles. Bottom pictures show sample table and a vial of $40 \mathrm{~nm}$ size $\mathrm{NV}$ nanodiamonds dispersed in a deionized water.
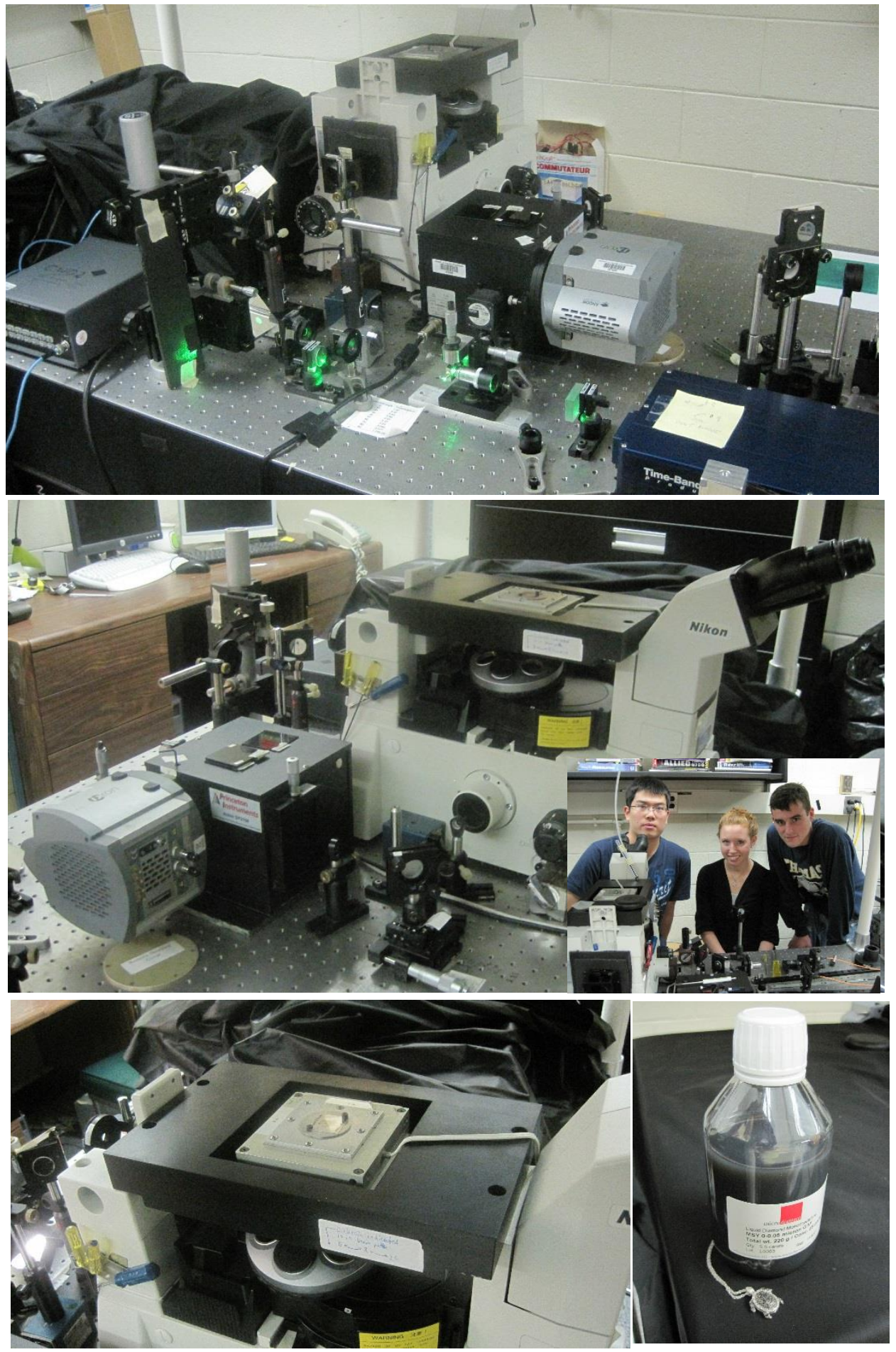

Figure 15. Pictures of a Labs 3 and 4 experimental setup at different view. TOP: A 532-nm excitation green laser is shown on the picture. CENTER: A spectrometer with a low-light level EM-CCD camera is shown. Insert shows students of the OPT 253 class. BOTTOM: A sample table with a piezo-electric scanning stage for a raster scan of the sample (left) and a vial with dispersion in a deionized water of NV-color-center nanodiamonds. For comparison a jewelry with color center rough diamond powder is shown as well. 
After fluorescence imaging of nanodiamonds students of the OPT 253 class obtain micrographs of nanodiamond topography (Figure 16, left top micrographs) using a compact atomic force microscope (Figure 16, top right). Bottom left picture shows Fall 2016 students of OPT 253 working on an AFM.

Weekly 1 hour lectures about the labs, their main concepts, devices and optical components, lab materials and overview of modern advances of quantum nanophotonics are very important in the OPT 253 class (see Figure 16, bottom right). In addition to lab report submission and quizzes before and after each lab, students have two exams (MidTerm and Final). Graduate students must submit also individual essays about single and entangled photon sources and make a Power Point presentation at a special meeting with TAs (for 20 students in the OPT 253/OPT 453 class two TAs were involved).
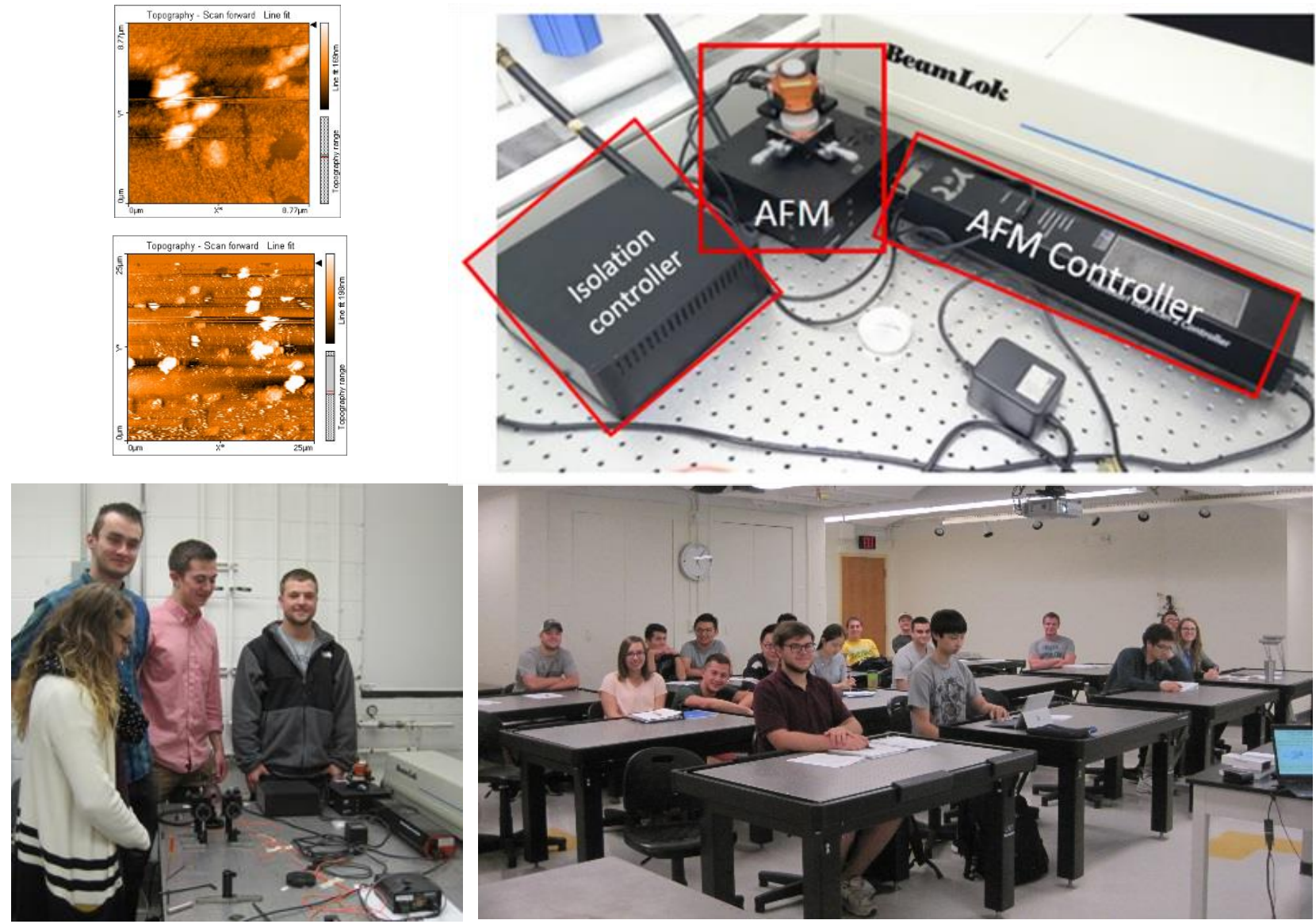

Figure 16. TOP LEFT: Raw data of AFM imaging of topography of nanodiamond clusters obtained by OPT 253 students; TOP RIGHT: A compact AFM (Easy Scan 2, Nanosurf Inc.) on a vibration isolation platform and a AFM controller. BOTTOM LEFT: Undergraduate students of a Fall 2016 OPT 253 class working on an AFM. BOTTOM RIGHT: Students of a Fall 2016 OPT 253/OPT 453 class on the lecture.

\subsection{Lab. 4. Single-photon source: Hanbury Brown and Twiss setup. Fluorescence antibunching}

To prove a single photon nature of single-emitter fluorescence (separation of all photons in time from the emitter (antibunching)), in a Lab 4 students measure time interval between consecutive photons. Antibunching first was obtained at the University of Rochester in 1977 by Mandel, Kimble and Dagenais [30-31]. See also book [32] and review [33].

In the case of antibunching should not be any photons at all at zero interphoton time. For such measurements a Hanbury Brown and Twiss correlator [34] is used (Figure 17). It consists on a beamsplitter and two single-photon counting avalanche photodiode modules APDs. Electronics part in our measurements consists of a time-correlated single photon counting PCI card with a start and stop inputs connected to APDs. This card (Time Harp 200, Picoquant) and a computer program permit to build a histogram of interphoton times. In the case of antibunching a dip appears at zero interphoton time. 
Figure 17, right shows user interface of the program used for building the histogram with a dip at zero photon time indicating antibunching. In these measurements in class we used a CdSeTe colloidal nanocrystal quantum dot (NQD) within a gold bow-tie nanoantenna. These 2015 OPT 253 class results were reported at 2016 Frontiers in Optics conference [35]. During collection data for the histogram, on another computer with imaging software students recorded time traces of changing intensities of single emitters (NQD) in time showing blinking of a single NQD (see Figure 17, insert).
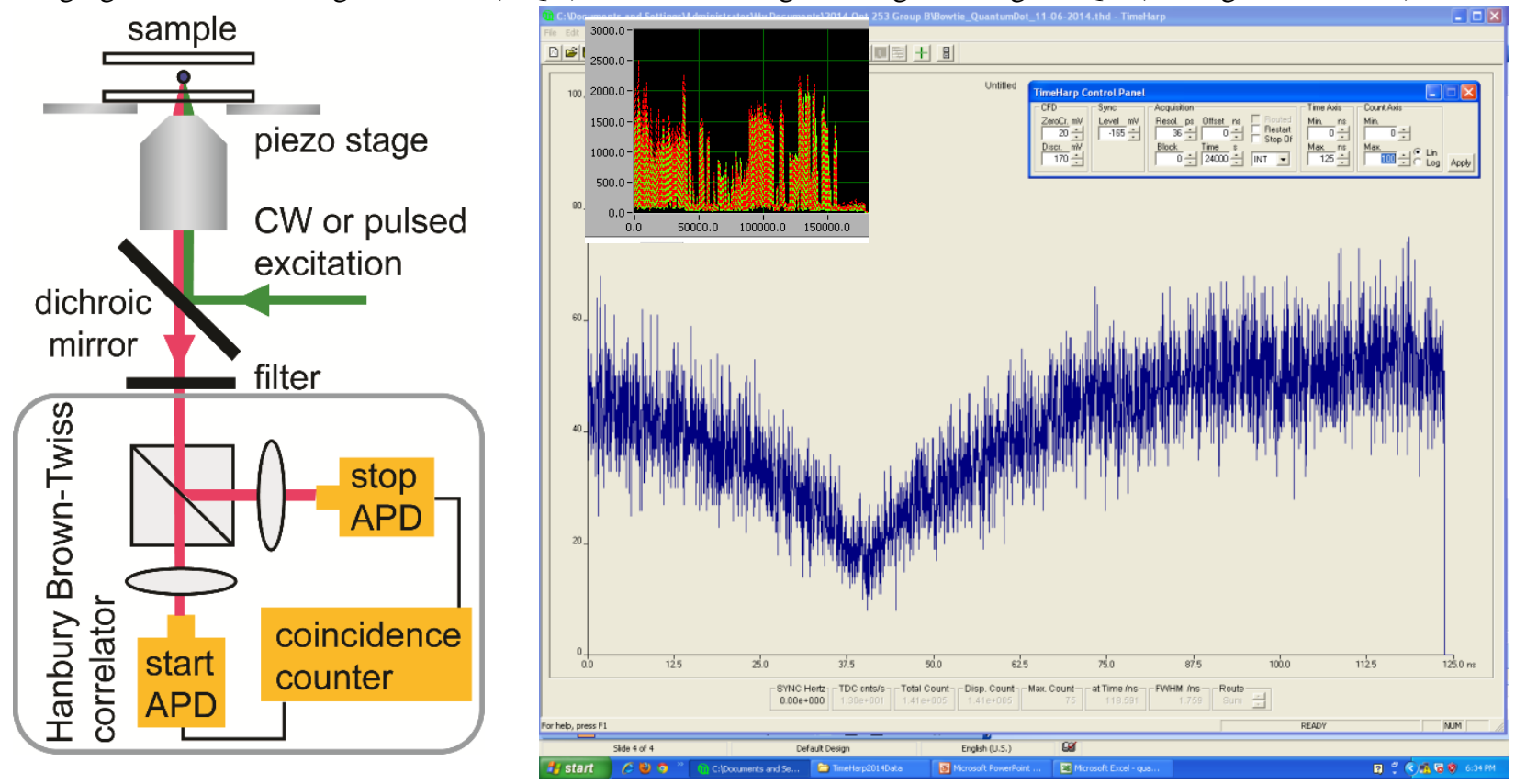

Figure 17. LEFT: Schematics of a Hanbury Brown and Twiss correlator at the ouput of a confocal fluorescence microscope (prepared by L. Bissell). RIGHT: Raw data histogram of interphoton times with a dip at zero time interval indicating photon antibunching (SPS). Fluorescence of NQD inside a gap of a bow-tie nanoantenna was studied. Insert shows a time trace of this NQD.

3 hours from this 15-hour lab (five 3 hour-classes for each group of 3-4 students) of the OPT 253/OPT 453 class were devoted to electronics. Students measured on an oscilloscope TTL pulses from APDs, they also calibrated a PCI correlator by finding a zero-time position on a histogram using an electrical delay line. BNC and PCI card connectors and cables and impedance matching are discussed as well. Detailed discussion was included on computer cards used in this lab.

\section{FRESHMAN PROJECTS, OPT 223, OPT 204, PHY 243W, AND PHY 534/OPT 554 CLASSES}

Starting from 2009, 12-15 hour quantum optics labs were introduced into a freshman course OPT 101 "Introduction to Optics" (W. Knox and T. Brown) as research projects. TAs (Ph.D students) of OPT 253 class assisted these projects. Sometimes with many freshmen selected "quantum" projects, additional TAs helped to supervise these projects. Figure 18 shows 3 groups of 16 freshmen of 2010 with 3 TAs who supervised freshmen projects on single (antibunched) photon source, entanglement and Bell's inequalities, and single photon interference.
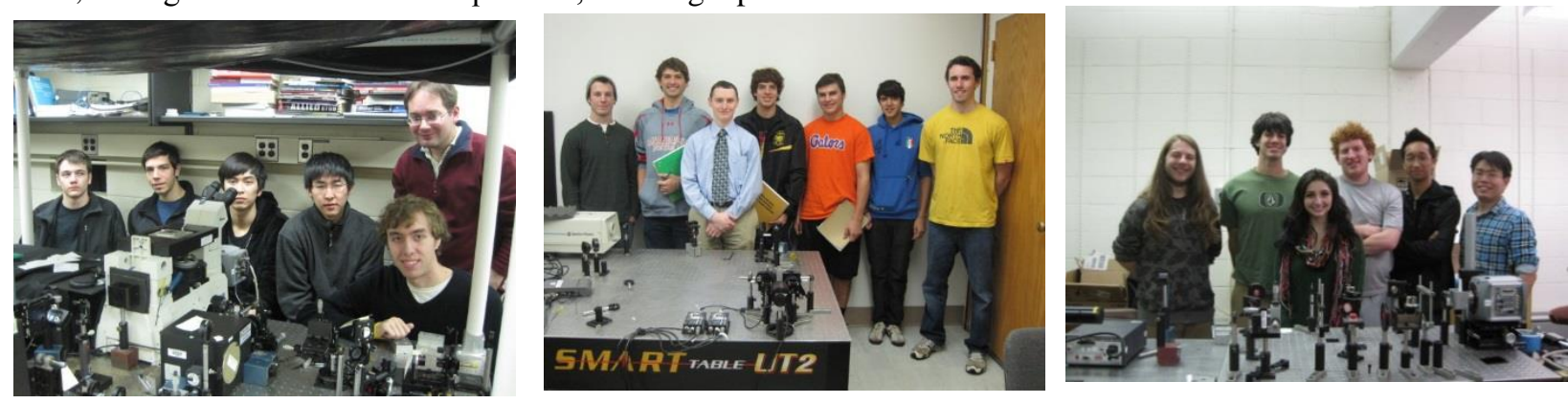

Figure 18. Three groups of freshmen of 2010 with their TAs on: LEFT: Single-photon source project (TA L. Bissell-right); CENTER: Entanglement and Bell's inequalities (TA G. Gehring - third from the left); RIGHT: Single photon interference (TA Z. Shi - right). 
From 2009 to 2012 all students of a required class OPT 223 "Quantum theory" (C. Stroud) carried out two 3 hour labs on entanglement and Bell's inequalities and single-photon interference (Figure 19, left). Students of a PHY 243 W “Advanced experimental techniques" (J. Howell) carried out 12-hour lab on entanglement (Figure 19, right). These labs were also part of a graduate course PHY 534/OPT 554 "Advanced topics on Quantum Optics" (M. Alonso, N. Bigelow, R. Boyd, J. Eberly, J. Howell, S. Lukishova, C. Stroud). At present time a single-photon interference 3-hour lab is a part of a required OPT 204 class "Sources and detectors labs and lab lectures" (S. Lukishova).
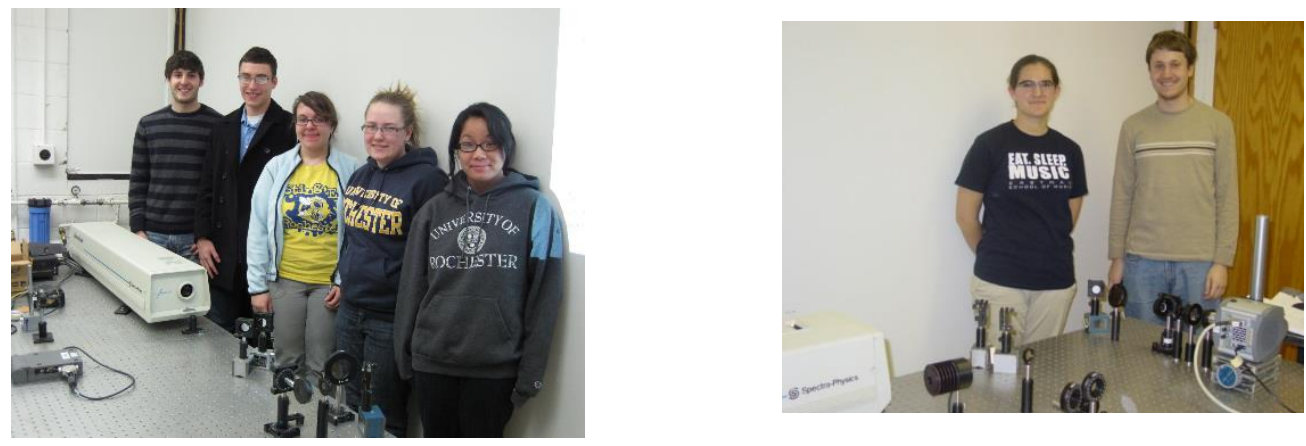

Figure 19. LEFT: OPT 223 class (2012) in entanglement and Bell's inequalities lab. RIGHT: PHY 243W students during an alignment of an EM-CCD camera to observe downconverted photon cones in entanglement and Bell's inequalities lab.

\section{TEACHING STUDENTS AND PROFESSORS FROM MONROE COMMUNITY COLLEGE AND OTHER COLLEGES AND UNIVERSITIES}

\subsection{Monroe Community College}

From 2009 to 2015 years, 142 MCC students carried out labs at the UR Quantum and Nano-Optics Lab facility. 52 MCC students from them also carried out the lab on photolithography in the UR Integrated Nanosystems Center (see paper [36] of this proceedings issue). MCC students carried out 3-hour labs on (1) entanglement and Bell's Inequalities; (2) singlephoton interference with single-photon counting EM-CCD camera; and (3) atomic force microscopy of nanodiamonds. Prof. P. D'Alessandris (MCC) was trained to work with an EM-CCD camera to teach his MCC students a single-photon interference lab at the UR (Figure 20). Prof. S. Lukishova taught MCC students other two labs. Within this collaboration supported by 3 NSF grants, lab manuals were specifically rewritten for MCC students [Lukishova (UR), D'Alessandris (MCC)]. Methods of evaluation of MCC students' knowledge were developed using quizzes with participation of an external evaluator (Prof. Zawicki, Buffalo State College).
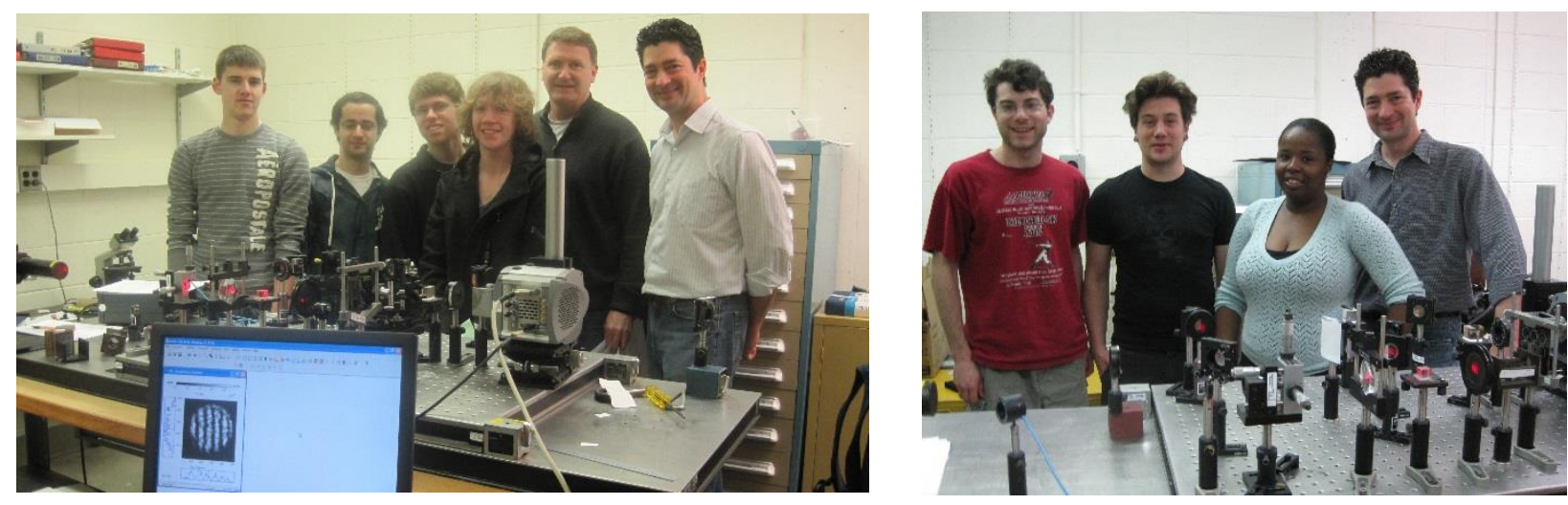

Figure 20. Two groups of MCC students with their professor (right) in the UR lab on single-photon interference.

\subsection{Rochester Institute of Technology}

Rochester Institute of Technology (RIT) was a collaborator of the UR on one of the NSF-supported projects on developing teaching experiments on photon quantum mechanics using photon counting instrumentation. During Fall 2009, Prof. R. 
Jodoin from RIT spent his sabbatical in UR teaching labs. Later RIT established their own quantum-optics teaching lab, strengthening the Upstate New York future-optics roots. Prof. S. Preble established quantum optics teaching lab on entanglement learning UR experience (see Figure 21).
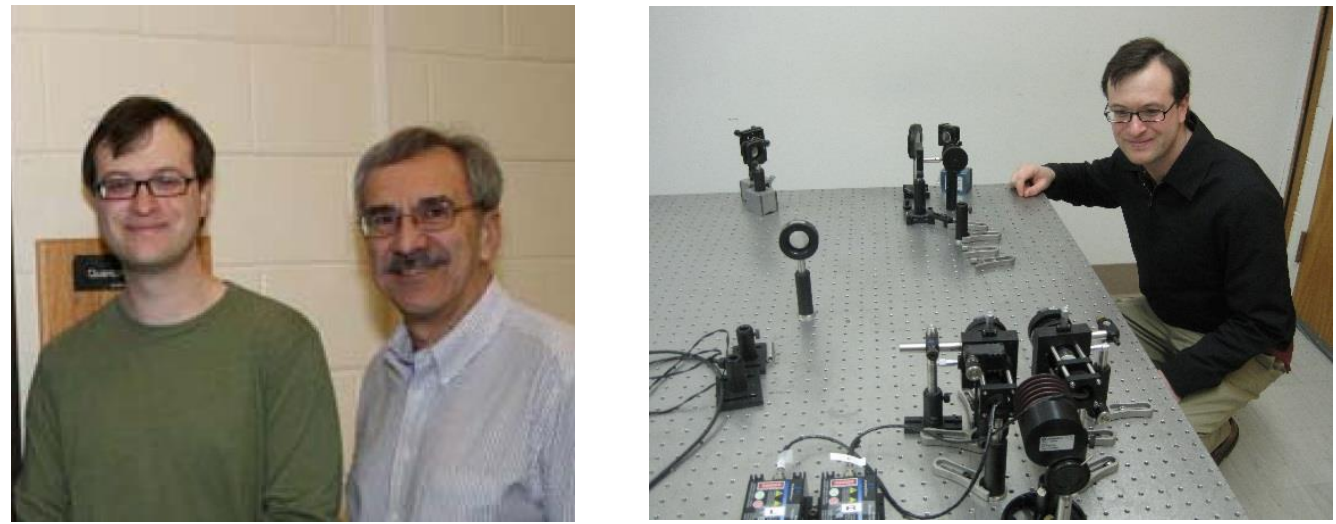

Figure 21. LEFT: S. Preble and R. Jodoin - RIT collaborators of UR. RIGHT: S. Preble at the UR facility learning alignment of entanglement and Bell's inequalities setup.

\subsection{UR participation at ALPhA's Laboratory Immersions Program}

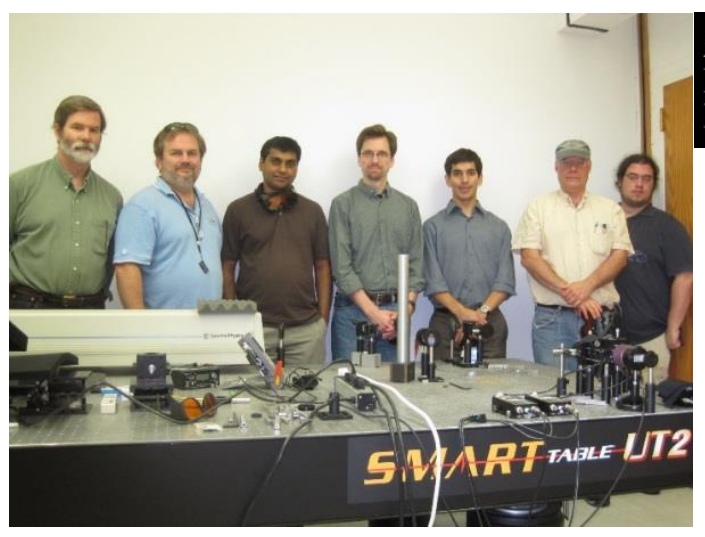

Figure 22. Six participants of the ALPhA's immersion program at the UR and a TA J. Winkler (right).

\subsection{Adelphi University students' visit for Quantum Lab experience}
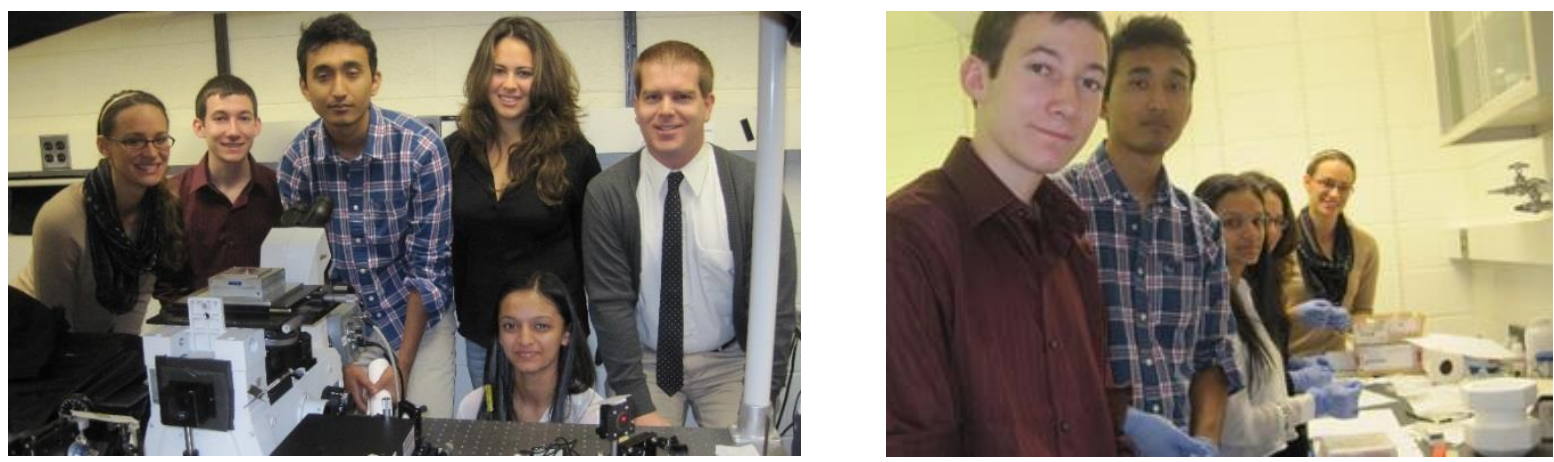

Figure 23. Adelphi university students and their professor at the UR Quantum and Nano-Optics Lab facility. LEFT: During their experiment on SPS (single-molecule confocal fluorescence microscopy of colloidal nanocrystal quantum dots). Prof. S. Bentley - at right. RIGHT: During their preparation of 1-D photonic band gap structures for SPS with a cholesteric liquid crystal microcavity [37]. 
During two days (October 2012), 5 students of Adelphi University carried out 4 labs at the UR Quantum/Nano-Optics Lab facility. They also prepared 1D-photonics bandgap materials for SPS based on cholesteric liquid crystals [37] (Figure 23).

\subsection{Dissemination of results in some other universities}

One graduate student of Prof. P. Verma (University of Oklahoma (UO), Tulsa) was trained on a Rochester lab facility in October 2010. Earlier (in September of 2010) S. Lukishova was invited to Tulsa to share her experience on quantum optics labs with this University. Figure 24, left shows UO audience at the Tulsa Schusterman Center Seminar during this lecture. Insert shows P. Verma with graduate student and S. Lukishova.
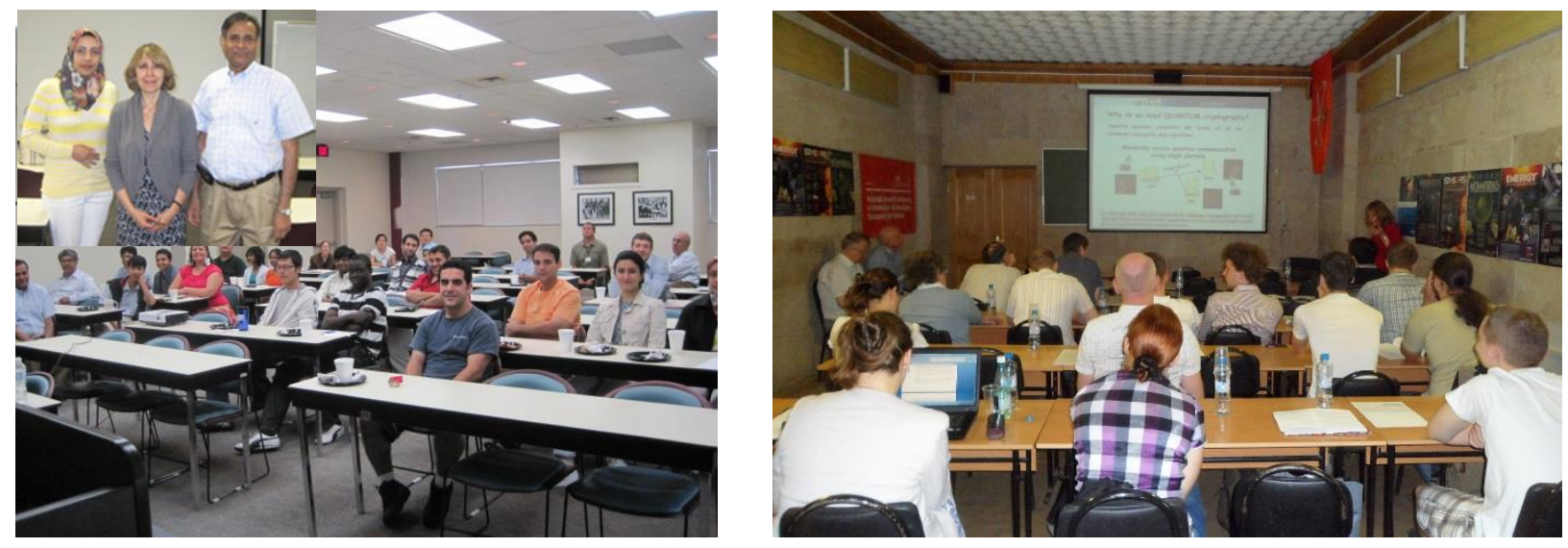

Figure 24. LEFT: Dissemination of results at the UO, Tulsa: lecture of S. Lukishova at the Tulsa Schusterman Center Seminar. Insert shows (from right to left) P. Verma, S. Lukishova and Verma's graduate student. RIGHT: Invited lecture of S. Lukishova at the ITMO University, St. Petersburg, Russia.

S. Lukishova was also invited to the Young Scientists Workshop at ITMO University in June 2013 (Saint Petersburg, Russia). See Figure24, right.

In Spring 2012, 6 graduate students and Prof. S.K. Sundaram (SUNY Alfred) visited UR Quantum and Nano-Optics Lab facility and attended lecture-demonstrations (Figure 25).

During several years students of Colgate University (Prof. E. Galvez) attended similar lecture demonstrations at the UR.

Figure 25. Students of Alfred University and their professor S.K. Sundaram at single-photon interference lab of the UR.

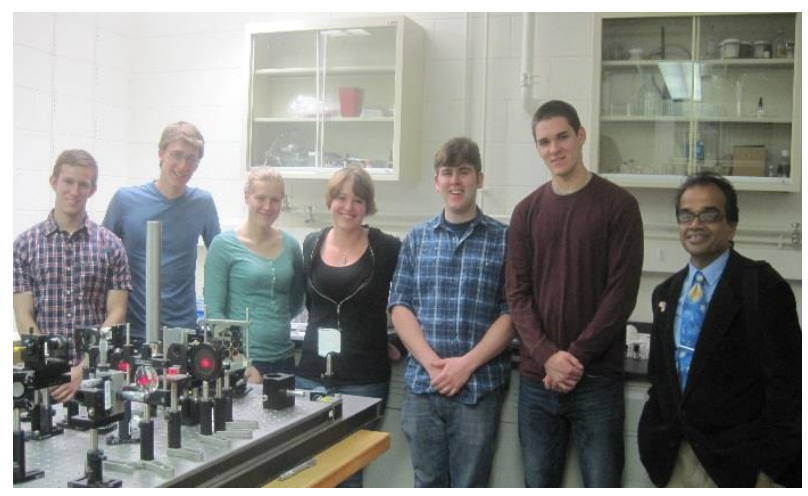

\section{ASSESSMENT METHODS}

NSF support requires evaluation of students learning with description of assessment methods. Here we provide only some examples of this work [17]. Multiple forms of assessment methods varied depending of a particular student group and the time students spent in the lab (3-15 hours): writing individual/group lab reports and essays, preparing individual/group presentations, exams with detailed answering 7-36 questions, etc. For the full 4-credit-hour course, performance assessment methods were also used, e.g., debating a topic, demonstrating a skill, presenting the laboratory notebooks. During the discussion with each student, students were asked to defend their scientific arguments because some questions permitted different types of answers. To recognize and analyze alternative explanations and models students were asked to write the essays on alternative technologies of single/entangled photon sources. For communication skills development sometimes students were divided into groups of two or three students. Maximum student number in the group was six.

Ungraded written and oral practice Mid term and Final exams for the full four-credit-hour course significantly contributed to the project's success. Technical problems have been worked out after practice exams during special lectures. Started 
from 2010 to 2014, ungraded pre-tests were also used before each lab after reading lab manuals, and at the end of each lab. With increasing a number of students in the class practice ungraded exams were excluded in spite of their effectiveness.

Some assessment methods measured meaningful learning outcomes, e.g., one of criteria of project success in 2009-2012 was that $80 \%$ of students of 4 -credit-hour course should have correct answers for $70 \%$ of the questions. The best criteria of success for freshmen, community college and undergraduate students of courses with three-hour ("mini") labs are still under development. For these groups of students we were investigated student capability to learn difficult concepts in a restricted time frame. Before the lab sessions, the lectures and discussions devoted to lab contents were included in all courses which incorporate two-3-hour lab sessions.

Learning outcomes describe what students are able to demonstrate in terms of knowledge and skills upon completion of the full lab course or the lab. We have three learning measurable outcomes: (a) students are able to demonstrate knowledge of the concepts of entanglement, quantum superposition and interference, wave-particle duality, single photons; (b) students demonstrate mastery in photon-counting instrumentation; (c) students are involved in research, combining research and education.

Teaching assistants help in summative evaluation. For instance, using a questionnaire with 36 questions on photon quantum mechanics in 2008, showed that one half students of 4-credit hour course answered correctly more than $75 \%$ of questions, $70 \%$ of students answered correctly more than $70 \%$ of questions and all students answered correctly more than $60 \%$ of questions. 2009 questionnaire with 32 questions showed that percent of correct answers of all students was greater than $90 \%$, in 2010 - greater than $85 \%$. This extraordinary success rate for quite difficult material effectively demonstrate the effectiveness of the laboratory approach.

Regarding 3-hour lab versions and freshmen involvement in the project, the results of summative evaluation using questionnaires with several questions are presented in histograms (Figures 26-27). Left parts of the figures are for 2008year and right parts are for 2009-year results. Histograms in Figure 26 show percent of correct answers for Lab 1 and Lab 2 (OPT 223 for seniors) after lab completion. Figure 27 shows the same histograms for MCC students.

OPT 223 students (16 and 20 students total)
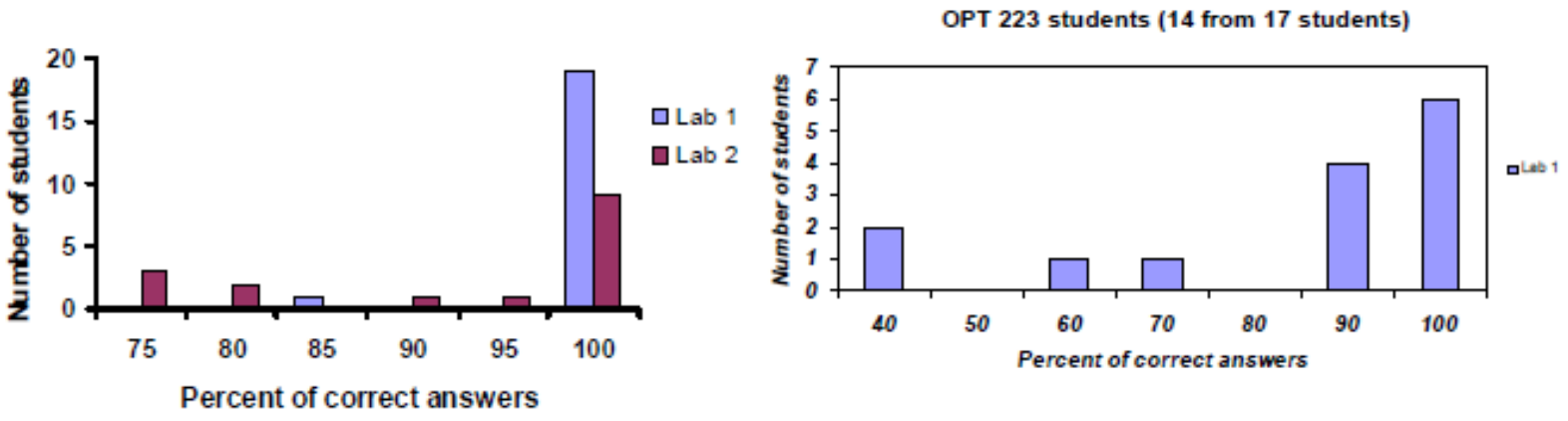

Figure 26. Histograms of evaluation of senior knowledge after lab completion (University of Rochester, Department of Optics, Quantum mechanics course OPT 223): LEFT- for 2008 year, RIGHT - for 2009 year.
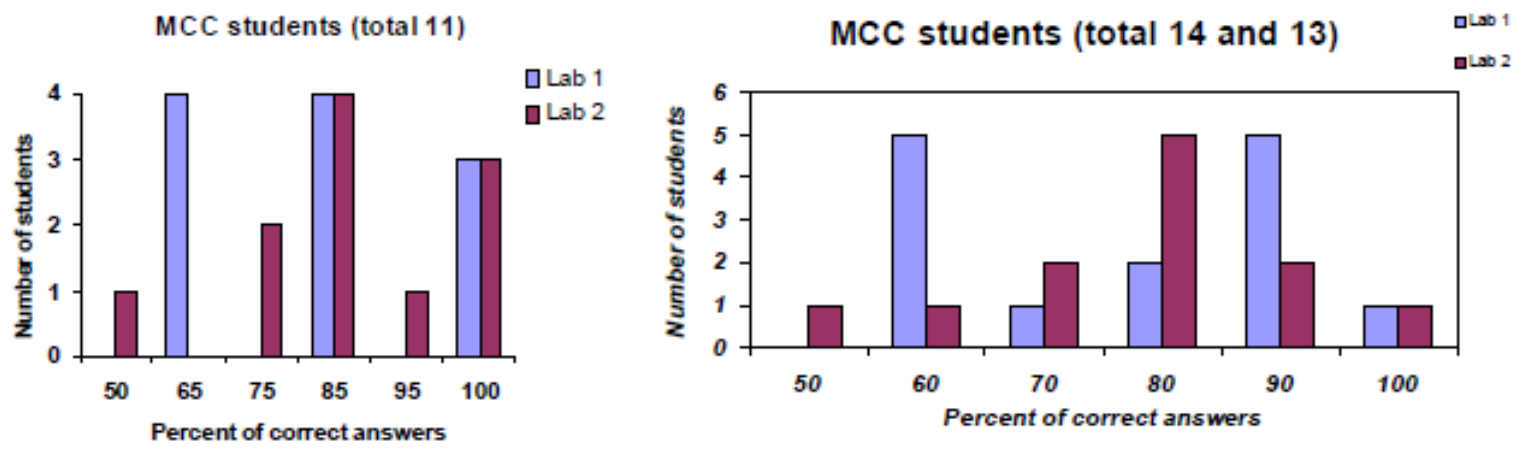

Figure 27. Histograms of evaluation of Rochester Monroe Community College student knowledge after lab completion (Modern Physics course): LEFT - for 2008 year, RIGHT - for 2009 year. 


\section{CONCLUSION}

The appearance of the new fields of quantum optics, quantum computation, and quantum communications and the rapid progress in photon-counting instrumentation open new opportunities for teaching the most difficult concepts of quantum mechanics by set of simple, easy understandable, and exciting experiments with single and entangled photons. Four labs were prepared at the Institute of Optics, University of Rochester on generation and characterization of entangled and single photons demonstrating the laws of quantum mechanics: (1) entanglement and Bell's inequalities, (2) single-photon interference (Young's double slit experiment and Mach-Zehnder interferometer), (3) confocal microscope imaging of single-emitter fluorescence, (4) Hanbury Brown and Twiss setup. Fluorescence antibunching.

We have adapted to the main challenge (the lack of space in the curriculum) by developing a series of modular 3-hour experiments and 20-min-demonstrations based on technical elective, 4-credit-hour laboratory course, that were incorporated into a number of courses ranging from freshman to senior level. Rochester Monroe Community College students also benefited from this facility. We also collaborated in this project, student assessment methods and criteria of success for different groups of diverse students, with Rochester Institute of Technology and Adelphi University. We participated in Immersion Program of Advanced Laboratory Physics Association by hosting 6 participants of this program and teaching them our experience in this field.

\section{ACKNOWLEDGEMENTS}

The author acknowledges the support by the NSF Awards ECS-0420888, DUE-0633621, DUE-0920500, EEC-1343673, the University of Rochester Kauffman Foundation Initiative, Wadsworth C. Sykes Faculty Engineering Award, the Hajim School of Engineering \& Applied Sciences (University of Rochester), and the Spectra-Physics division of Newport Corporation. The author also thanks C.R. Stroud for constant support and collaboration, P. D'Alessandris for collaboration, W. Knox and T. Brown for collaboration with freshmen projects, P. Adamson for his help and support, and J. Choi and A.K. Jha for their contributions to entanglement setups.

\section{REFERENCES}

[1] Lukishova, S.G., "Quantum Optics, Quantum Information and Nano-Optics Laboratory", http://www.optics.rochester.edu/workgroups/lukishova/QuantumOpticsLab/ .

[2] Einstein, A., Podolsky, B., Rosen, N., "Can quantum-mechanical description of physical reality be considered complete?", Phys. Rev. 47 (10), 777-780 (1935).

[3] Schrödinger, E., "Discussion of probability relations between separated systems," Proceedings of the Cambridge Philosophical Society 31, 555-563 (1935).

[4] Bell, J., "On the Einstein Podolsky Rosen paradox", Physics 1 (3), 195-200 (1964).

[5] Bell, J.S., [Speakable and Unspeakable in Quantum Mechanics: Collected Papers on Quantum Philosophy], introduction of Aspect A., Cambridge University Press, Cambridge, New York (2004).

[6] Eberly, J.H., "Bell inequalities and quantum mechanics", Am. J. Phys. 70 (3), 276-279 (2002).

[7] Clauser, J.F., Horne, M.A., Shimony A., Holt, R.A., "Proposed experiment to test local hidden-variable theories", Phys. Rev. Lett., 23, 880-884 (1969).

[8] Kwiat, P.G., Waks, E., White, A.G., Appelbaum, I., Eberhard, P.H., "Ultrabright source of polarization-entangled photons", Phys. Rev. A. 60, R773-R776 (1999).

[9] Dehlinger, D. and Mitchell, M.W., "Entangled photon apparatus for the undergraduate laboratory", Am. J. Phys, 70, 898-902 (2002).

[10] Dehlinger, D. and Mitchell, M.W., "Entangled photons, nonlocality, and Bell inequalities in the undergraduate laboratory", Am. J. Phys, 70, 903-910 (2002).

[11] Galvez, E.J., "Resource Letter SPE-1: Single-Photon Experiments in the Undergraduate Laboratory," American Journal of Physics 82, 1018-1028 (2014).

[12] Galvez, E.J., "Qubit Quantum Mechanics with Correlated Photon Experiments," American Journal of Physics 78 510-519 (2010). 
[13] Galvez, E.J., Holbrow, C.H., Pysher, M.J., Martin, J.W. Courtemanche, N., Heilig, N.L., and Spencer, J., "Interference with correlated photons: Five quantum mechanics experiments for undergraduates," Am. J. of Phys. 73, 127-140 (2005).

[14] Thorn, J.J., Neel, M.S., Donato, V.W., Bergreen, G.S., R. E. Davies, R.E., and M. Beck, M. "Observing the quantum behavior of light in an undergraduate laboratory,"Am. J. Phys. 72, 1210-1219 (2004)

[15] Gogo, A., Snyder,W.D., and Beck, M., "Comparing quantum and classical correlations in a quantum eraser," Phys. Rev. A 71, 052103 (2005).

[16] Galvez, E.Z. and Beck, M., "Quantum optics experiments with single photons for undergraduate laboratories", in Education and Training in Optics 2007, edited by Nantel, M. (SPIE Digital Library, 2007), pp. 1-8 [http://spie.org/x102158.xml].

[17] Lukishova, S.G.,"Quantum optics laboratory for the undergraduate curriculum: teaching quantum mechanics with photon counting equipment", S.G. Lukishova, 6 pages, (http://stl.asee.org/papers_2011/Lukishova.pdf), Proceedings of the American Society for Engineering Education (ASEE), St-Lawrence Chapter Annual Conference, 18-19 March 2011, Excelsior College, Albany NY (2011).

[18] Beck, M., [Quantum Mechanics. Theory and Experiment], Oxford University Press, Oxford, NY (2012).

[19] Prutchi, D. and Prutchi, S.R., [Exploring Quantum Physics through Hands-on-Projects], Wiley, New Jersey (2012).

[20] Galvez, E. K., "Photon Quantum Mechanics", http://departments.colgate.edu/physics/research/Photon/root/photon_quantum_mechanics.htm .

[21] Beck, M., "Modern Undergraduate Quantum Mechanics Experiments", http://people.whitman.edu/ beckmk/QM/ .

[22] Rae, A., [Quantum Physics: Illusion or Reality], Cambridge Univ. Press, Cambridge, London, NY (1986).

[23] Fox, M., [Quantum Optics. An Introduction], Oxford University Press, Oxford, NY (2007).

[24] Gerry, C.C. and Knight, P.L, [Introductory Quantum Optics], Cambridge Univ. Press, Cambridge, NY (2005).

[25] Walls, D.F. and Milburn, G.J., [Quantum Optics], Springer, Berlin, Heidelberg, NY (1995).

[26] Bachor, H.-A., [A Guide to Experiments in Quantum Optics], Wiley-VCH, Weinheim, NY (1998).

[27] Greenstein, G. and Zajonc, A.G., [The Quantum Challenge. Modern Research on the Foundations of Quantum Mechanics], Jones and Bartlett, Boston, Toronto (2006).

[28] Klyshko, D.N., [Photons and Nonlinear Optics], Gordon and Breach, NY (1988).

[29] Schneider M.B., and LaPuma, I.A., "A simple experiment for discussion of quantum interference and which-way measurements", Am. J. Phys. 70 (3), 266 (2002).

[30] Kimble, H.J., Dagenais, M., and Mandel, L., "Photon antibunching in resonance fluorescence”, Phys. Rev. Lett. 39, 691-695 (1977).

[31] Walls, D.F., "Evidence for the quantum nature of light", Nature 280, 451-454 (1979).

[32] Migdall, A., Polyakov, S.V., Fan, J., Bienfang, J.C., (eds.), [Single-photon generation and detection], Elsevier (2013).

[33] Lukishova, S.G. and Bissell, L.J., "Nanophotonic advances for room-temperature single-photon sources", in [Advances in Quantum Photonics: from the First Single-photon and Nonlinear Optical Experiments to Modern Quantum Photonics], Boyd, R.W., Lukishova, S.G. and Zadkov, V., Eds., Springer, Berlin, NY (2017).

[34] Hanbury Brown, R., and Twiss, R.Q., "Correlation between photons in two coherent beams of light", Nature 177, 27-29 (1956).

[35] Lukishova, S.G., Mihaylova, D., Zhu, H., Liapis, A., Boyd, R.W., "Plasmonic bowtie nanoantennas with nanocrystal quantum dots for single-photon source applications", Frontiers in Optics 2016, OSA Technical Digest, (Optical Society of America, Rochester NY, September 17-21, 2016), paper LF2D.6, https://doi.org/10.1364/LS.2016.LF2D.6.

[36] Lukishova, S.G., Bigelow, N.P., D'Alessandris, P.D., "Development of multidisciplinary nanotechnology undergraduate education program at the University of Rochester Integrated Nanosystems Center", paper 100-184, 14th International Conference on Education and Training in Optics \& Photonics (ETOP), May 29-31, 2017, Hangzhou, China.

[37] Lukishova, S.G., "Liquid crystals under two extremes: (1) high-power laser irradiation, and (2) single-photon level", Molecular Crystals and Liquid Crystals, 559, Special Issue, 127-157 (2012). 